\title{
Enhanced cGAS-STING-dependent interferon signaling associated with mutations in ATAD3A
}

\author{
Alice Lepelley ${ }^{1}$ (D), Erika Della Mina ${ }^{1}$ (D), Erika Van Nieuwenhove ${ }^{2,3,4}$ (D), Lise Waumans ${ }^{5}$ (D), Sylvie Fraitag ${ }^{6}$ (D), Gillian I. Rice ${ }^{7}$, Ashish Dhir ${ }^{8}$ (D),

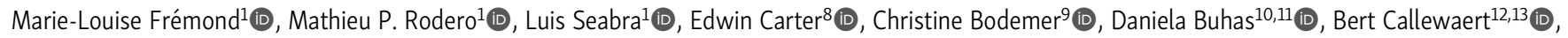 \\ Pascale de Lonlay ${ }^{14,15}$ (D), Lien De Somer ${ }^{16,17,18}$ (D), David A. Dyment ${ }^{19,20}$ (D), Fran Faes ${ }^{21}$ (D), Lucy Grove (D), Simon Holden $^{23}$ (D), Marie Hully ${ }^{24}$ (D), \\ Manju A. Kurian ${ }^{25}$ (D) , Hugh J. McMillan ${ }^{26}$ (D), Kristin Suetens ${ }^{27,28}$ (D), Henna Tyynismaa ${ }^{29}$ (D), Stéphanie Chhun ${ }^{30,31,32}$ (D), Timothy Wai ${ }^{33}$ (D), \\ Carine Wouters ${ }^{16,17,18}$ (D), Brigitte Bader-Meunier ${ }^{34}$ (D), and Yanick J. Crow ${ }^{1,8}$ (D)
}

\begin{abstract}
Mitochondrial DNA (mtDNA) has been suggested to drive immune system activation, but the induction of interferon signaling by mtDNA has not been demonstrated in a Mendelian mitochondrial disease. We initially ascertained two patients, one with a purely neurological phenotype and one with features suggestive of systemic sclerosis in a syndromic context, and found them both to demonstrate enhanced interferon-stimulated gene (ISC) expression in blood. We determined each to harbor a previously described de novo dominant-negative heterozygous mutation in ATAD3A, encoding ATPase family AAA domain-containing protein $3 A$ (ATAD3A). We identified five further patients with mutations in ATAD3A and recorded upregulated ISG expression and interferon a protein in four of them. Knockdown of ATAD3A in THP-1 cells resulted in increased interferon signaling, mediated by cyclic GMP-AMP synthase (cGAS) and stimulator of interferon genes (STING). Enhanced interferon signaling was abrogated in THP-1 cells and patient fibroblasts depleted of mtDNA. Thus, mutations in the mitochondrial membrane protein ATAD3A define a novel type I interferonopathy.
\end{abstract}

\footnotetext{
IUniversité de Paris, Imagine Institute, Laboratory of Neurogenetics and Neuroinflammation, Institut National de la Santé et de la Recherche Médicale, Unité mixte de recherche 1163, Paris, France; ${ }^{2}$ Universitair Ziekenhuis Leuven, Department of Pediatrics, Leuven, Belgium; ${ }^{3}$ Department of Microbiology and Immunology, Laboratory of Adaptive Immunity, Katholieke Universiteit Leuven, Leuven, Belgium; ${ }^{4} \mathrm{VIB}-\mathrm{KU}$ Leuven Center for Brain and Disease Research, Leuven, Belgium; ${ }^{5}$ Department of Pathology, Universitair Ziekenhuis Leuven, Campus Gasthuisberg, Leuven, Belgium; ' ${ }^{6}$ Service d'Anatomo-Pathologie, Hôpital Necker-Enfants-Malades, Assistance Publique - Hôpitaux de Paris, Paris, France; 'Division of Evolution and Genomic Sciences, School of Biological Sciences, Faculty of Biology, Medicine and Health, University of Manchester, Manchester Academic Health Science Centre, Manchester, UK; ${ }^{8}$ Medical Research Council Human Genetics Unit, Institute of Genetics and Cancer, University of Edinburgh, Edinburgh, UK; ${ }^{9}$ Department of Dermatology and Reference Centre for Genodermatoses and Rare Skin Diseases, Imagine Institute, Hôpital Universitaire Necker-Enfants Malades, Assistance Publique - Hôpitaux de Paris, Université Paris-Centre, Paris, France; ${ }^{10}$ Medical Genetics Division, Department of Specialized Medicine, McGill University Health Centre, Montreal, Canada; ${ }^{11}$ Human Genetics Department, McGill University, Montreal, Quebec, Canada; ${ }^{12}$ Center for Medical Genetics, Ghent University Hospital, Ghent, Belgium; ${ }^{13}$ Department of Biomolecular Medicine, Ghent University, Ghent, Belgium; ${ }^{14}$ Reference Center for Inherited Metabolic Diseases, Necker Hospital, Assistance Publique - Hôpitaux de Paris, Institut National de la Santé et de la Recherche Médicale U1151, Institut Necker Enfants Malades, Université de Paris, Filière G2M, MetabERN, Paris, France; ${ }^{15}$ Institut Imagine, Institut National de la Santé et de la Recherche Médicale Unité mixte de recherche 1163, Paris, France; ${ }^{16}$ Pediatric Rheumatology, Universitair Ziekenhuis Leuven, Leuven, Belgium; ${ }^{17}$ Laboratory of Immunobiology, Rega Institute, Katholieke Universiteit Leuven, Leuven, Belgium; ${ }^{18}$ European Reference Network for Rare Immunodeficiency, Autoinflammatory and Autoimmune Diseases at University Hospital Leuven, Leuven, Belgium; ${ }^{19}$ Department of Genetics, Children's Hospital of Eastern Ontario, Ottawa, Ontario, Canada; ${ }^{20}$ Children's Hospital of Eastern Ontario Research Institute, Ottawa, Ontario, Canada; ${ }^{21}$ Department of Pediatric Neurology, Ghent University Hospital, Ghent, Belgium; ${ }^{22}$ Community Paediatric Department, West Suffolk Hospital Foundation Trust, Bury St Edmunds, UK; ${ }^{23}$ Department of Clinical Genetics, Addenbrooke's Hospital, Cambridge, UK; ${ }^{24}$ Pediatric Neurology Department, Hôpital Necker-Enfants Malades, Assistance Publique - Hôpitaux de Paris, Paris, France; ${ }^{25}$ Developmental Neurosciences, University College London Great Ormond Street Institute of Child Health, London, UK; ${ }^{26}$ Children's Hospital of Eastern Ontario Research Institute, University of Ottawa, Ottawa, Canada; ${ }^{27}$ Department of Radiology, University Hospitals Leuven, Radiology, Leuven, Belgium; ${ }^{28}$ Department of Radiology, Regional Hospital Heilig Hart Leuven, Leuven, Belgium; ${ }^{29}$ Stem Cells and Metabolism Research Program, Faculty of Medicine and Neuroscience Center, Helsinki Institute of Life Science, University of Helsinki, Helsinki, Finland; ${ }^{30}$ Paris Descartes University, Université de Paris, Sorbonne-Paris-Cité, Paris, France; ${ }^{31}$ Laboratory of Immunology, Hôpital Necker-Enfants Malades, Assistance Publique-Hôpitaux de Paris, Centre-Université de Paris, Paris, France; ${ }^{32}$ Institut Necker-Enfants Malades, Centre National de la Recherche Scientifique Unité mixte de recherche 8253, Institut National de la Santé et de la Recherche Médicale Unité mixte de recherche 1151, Team Immunoregulation and Immunopathology, Paris, France; ${ }^{3}$ Mitochondrial Biology Group, Institut Pasteur, Centre National de la Recherche Scientifique, Unité mixte de recherche 3691, Paris, France; ${ }^{34}$ Pediatric Immunology-Hematology and Rheumatology Unit, Hôpital Necker-Enfants Malades, Laboratory of Immunogenetics of Pediatric Autoimmunity, Institut National de la Santé et de la Recherche Médicale Unité mixte de recherche 1163, Assistance Publique - Hôpitaux de Paris, Institut Imagine, Paris, France.
}

Correspondence to Yanick J. Crow: yanickcrow@mac.com.

(c) 2021 Lepelley et al. This article is distributed under the terms of an Attribution-Noncommercial-Share Alike-No Mirror Sites license for the first six months after the publication date (see http://www.rupress.org/terms/). After six months it is available under a Creative Commons License (Attribution-Noncommercial-Share Alike 4.0 International license, as described at https://creativecommons.org/licenses/by-nc-sa/4.0/). 


\section{Introduction}

The type I interferonopathies are Mendelian inborn errors of immunity in which type I interferon (IFN) signaling is enhanced and considered directly relevant to disease causation (Crow and Manel, 2015). Definition of this grouping has highlighted the risks to human health of the sensing of self-derived nucleic acids, leading to the induction of an inappropriate type I IFNmediated antiviral response (Uggenti et al., 2019). IFN has also been implicated in the pathology of a variety of complex, autoimmune disorders (Muskardin and Niewold, 2018). Mitochondria represent vestigial bacteria living in the cytosol of eukaryotic cells, and while they play a central role in oxidative phosphorylation, metabolism, and apoptosis, there is increasing recognition of the potential of mitochondrial-derived nucleic acid to act as an agonist of the IFN signaling machinery in the context of disease (Dhir et al., 2018; Kim et al., 2019; Yu et al., 2020).

Here, we report dominant-negative mutations in ATAD3A, a ubiquitously expressed mitochondrial protein, to cause mitochondrial DNA (mtDNA)-dependent up-regulation of type I IFN signaling. ATAD3A has been described to have pleiotropic functions, including in mtDNA maintenance, mitophagy dynamics, and steroid synthesis (Baudier, 2018), none of which have been directly linked to type I IFN signaling. As a member of the AAA ATPase family, the protein has been suggested to form oligomers required for processive ATP hydrolysis within the oligomer (Gilquin et al., 2010), so that ATAD3A variants can act in a dominant-negative manner in the presence of WT ATAD3A by interfering with oligomerization or ATPase activity (Cooper et al., 2017; Frazier et al., 2021). While previously described patients with mutations in ATAD3A were ascertained on the basis of neurological involvement, two of the individuals that we report demonstrate features of systemic sclerosis, an autoimmune connective tissue phenotype where a disturbance of type I IFN signaling has been suggested to play an important pathogenic role (Skaug and Assassi, 2020).

\section{Results and discussion}

\section{Description of patients, their mutational status, and ex vivo IFN signaling}

As part of an ongoing protocol involving the agnostic screening of patients with uncharacterized phenotypes for up-regulation of type I IFN signaling, we identified two individuals demonstrating persistently enhanced IFN-stimulated gene (ISG) expression in their blood.

Patient 1 exhibits purely neurological disease (see Materials and methods for full clinical details). Now aged $7 \mathrm{yr}$, she is profoundly developmentally delayed and centrally hypotonic, with a peripheral dystonia and no useful motor function (Table S1). Communication is limited to eye movements and facial expression. In light of the prominent dystonia and a raised level of neopterin in cerebrospinal fluid (CSF) suggestive of an inflammatory process, ISG expression was assessed in blood and found to be markedly elevated on each of four occasions tested between the ages of 2 and $9 \mathrm{yr}$. Trio exome sequencing identified a previously described heterozygous c.1582C $>$ T (p.(Arg528Trp); R528W) de novo mutation in ATAD3A (Harel et al., 2016).
Patient 2 attended university with no intellectual deficits but demonstrates a spastic diplegic gait that appears stable since early infancy. She was diagnosed with antibody-positive Hashimoto's thyroiditis, vitiligo, and growth hormone deficiency in childhood (Table S1). Beginning at 21 yr of age, she developed sclerodermatous involvement of the hands, face, and ventral surface of the forearms, which progressed rapidly over a few months (Rodnan skin score: 33/51), fulfilling criteria for a diagnosis of systemic sclerosis (van den Hoogen et al., 2013; Fig. S1 A). Skin biopsy of affected tissue showed classical features of systemic sclerosis (Fig. S1 B). Immunoglobulin levels and antinuclear and anti-double-stranded DNA (anti-dsDNA) antibody titers were persistently elevated, but systemic sclerosis-related antibodies were not detected (Table S2). Continued clinical progression despite oral corticosteroids led to the introduction of rapamycin, which was associated with a partial regression of the cutaneous fibrosis and a decrease of the Rodnan skin score to 10/51 over 2 yr. ISG expression in blood was markedly elevated on each of seven occasions tested between the ages of 22 and 24 yr. Trio exome sequencing identified a previously described heterozygous c.1064G>A (p.(Gly355Asp); G355D) de novo mutation in ATAD3A (Cooper et al., 2017).

Given the above findings, we went on to ascertain other patients known to harbor mutations in ATAD3A. In this way, we recruited a further five individuals (Table $S 1$ and Materials and methods).

Patient 3 demonstrated early-onset severe motor delay with impaired cognition but good social contact. From age 2 yr, she developed progressive hardening of the skin. At $4 \mathrm{yr}$ of age, she exhibited perioral sclerosis, tight sclerodermatous skin of the upper and lower limbs, and severe flexion contractures at the knees, ankles, elbows, wrists, and fingers (Rodnan skin score: 32/51; Fig. S1 A). Skin biopsy revealed features typical of systemic sclerosis (Fig. S1 B). Magnetic resonance imaging (MRI) of the upper limbs confirmed cutaneous thickening (Fig. S1 C). Treatment with rapamycin and low-dose corticosteroids was associated with a good clinical response. She was shown to carry the same p.(Arg528Trp) substitution recorded in patient 1 . She demonstrated enhanced ISG expression in blood on the single occasion tested at age $4 \mathrm{yr}$.

Patients 4 and 5, both males, exhibit severe global developmental delay, hypertrophic cardiomyopathy, and a peripheral neuropathy. Patient 5 , like patient 2 , has a history of vitiligo and growth hormone deficiency. Patients 4 and 5 carry the same p.(Arg528Trp) substitution in ATAD3A as seen patients 1 and 3, patient 5 having been previously included in the first published series of cases with mutations in ATAD3A (family 3 II-I in Harel et al., 2016). Both demonstrated enhanced expression of ISGs in blood (three and two times, respectively). Additionally, when assessed at the same time as ISG expression, elevated levels of IFN- $\alpha$ protein were recorded three times in the serum of patient 4.

As recently reported (Hanes et al., 2020), patient 6, a female, has severe neurological disease due to a homozygous pathogenic splice-site transition (c.528+3A>G) in ATAD3A. CSF IFN- $\alpha$ protein was highly elevated at $1 \mathrm{yr}$ of age, while blood ISG expression was normal on the one occasion tested at age $2 \mathrm{yr}$. Bilateral calcification of the basal ganglia was present on cranial computed tomography (CT) at this time. 
A

Patient 1

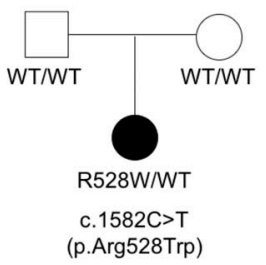

Patient 5

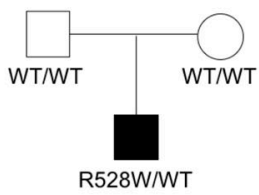

R528W/WT

(p.Arg528Trp)

B

$$
\begin{aligned}
& \text { H.sapiens } \\
& \text { G.gorilla } \\
& \text { P.abelii } \\
& \text { R.norvegicus } \\
& \text { M.musculus } \\
& \text { C.familiaris } \\
& \text { B.taurus } \\
& \text { G.gallus } \\
& \text { X.tropicalis } \\
& \text { T.nigroviridis } \\
& \text { G.morhua } \\
& \text { D.melanogaster } \\
& \text { C.elegans }
\end{aligned}
$$

Patient 3

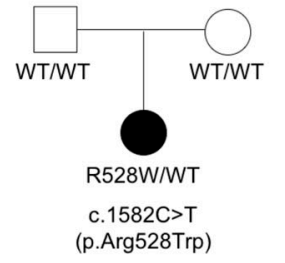

Patient 4

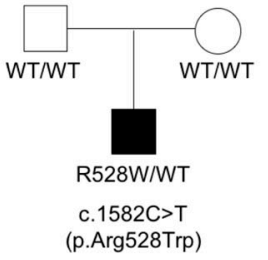

Patient 7

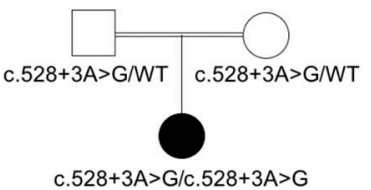

c. $528+3 A>G / c .528+3 A>G$

Gly355Asp NILMYGPPGTGKTLFAKKI HILLYGPPGTGKTLFAKKI NILMYGPPGTGKTLFAKKL NILMYGPPGTGKTLFAKKL
NVLMYGPPGTGKTLFAKKL NVLMYGPPGTGKTLFAKKL NILAYGPPGTGKTLFAKKI NVLMYGPPGTGKTLFAKKI NILMYGPPGTGKTLFAKKI NILMYGPPGTGKTLFAKKI NILMYGPPGTGKTLFAKKI NILMYGPPGTGKTLFAKKI NVLMHGPPGTGKTMFAKKI NVMFYGPPGTGKTLFAKSL

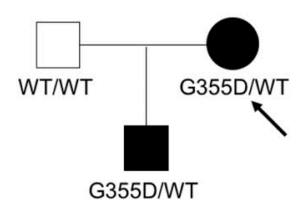

c. $1064 \mathrm{G}>\mathrm{A}$ (p.Gly355Asp)

Arg528Trp TEGMSGREIAQLAVSWQ TEGMSGREIAQLAVSWQ TEGMSGREIAQLAVSWQ TEGMSGREIAQLAVAWO TEGMSGREISOLAVAWO TEGMSGRE ISOLAVAWQ TEGMSGREISQLAVAWQ TEGMSGREISKLGVAWQ TEGMSGREISKLGVAWO TEGMSGREISKLGVAWO CEGMSGREISKLGVSWQ TSGMSGRELSKLVIGWQ $* * * * * *::: * \quad: . * *$ TAGMSGREIAQLAVAWO

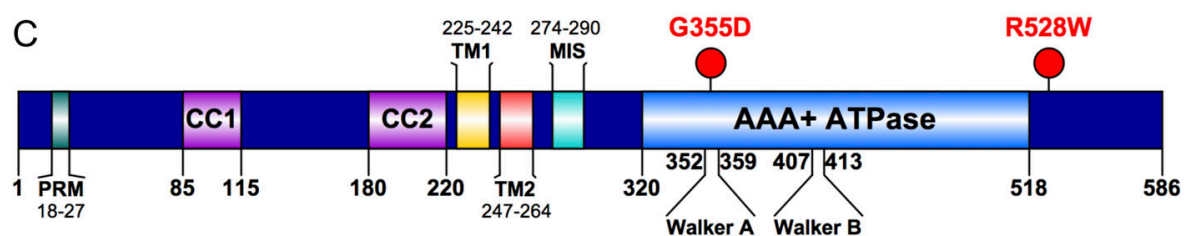

Patient 7 is of completely normal intellect into midadulthood. As previously identified by Cooper et al. (2017), she carries the same p.(Gly355Asp) mutation in ATAD3A as seen in patient 2. She was noted to demonstrate bilateral lower limb spasticity in early childhood. At age $40 \mathrm{yr}$, she exhibits severe lower limb spasticity and weakness. ISG expression was normal when assessed at this age. Although not tested here, her son, carrying the same mutation as his mother, has a severe dyskinetic motor disorder and profound intellectual delay.

\section{Summary of molecular data}

Six of seven patients ascertained in this study are heterozygous for one of two missense mutations (p.(Gly355Asp); p.(Arg528Trp)) in ATAD3A (Fig. $1 \mathrm{~A}$ ), neither of which is recorded on publicly available databases, being absent from $>250,000$ control alleles on gnomAD. The substituted amino acids are highly evolutionarily conserved (Fig. 1 B), and the amino acid substitutions are predicted as damaging by in silico algorithms (SIFT: damaging; Polyphen2: probably damaging). The glycine to aspartate substitution at amino acid 355 falls within the highly conserved Walker A motif, responsible for ATP binding in the AAA+-ATPase module of ATAD3A (Fig. 1 C; Baudier, 2018). Importantly, recombinant p.(Gly355Asp) mutant ATAD3A protein was shown to be associated with a markedly reduced ATPase activity and demonstrated a strong dominant-negative effect on the ATPase activity of WT protein (Cooper et al., 2017). Similarly, the Drosophila melanogaster equivalent of human p.(Arg528Trp) has also been documented to behave as a dominant-negative allele (Harel et al., 2016). Meanwhile, the homozygous c.528+3A $>G$ transition seen in patient 6 results in a significant reduction of ATAD3A protein in patient fibroblasts (Hanes et al., 2020).

\section{Summary of ex vivo IFN signaling}

The combination of neurological and immunological features is characteristic of diseases within the type I interferonopathy grouping, and patients with type I interferonopathies demonstrate high levels of ISG mRNA in whole blood. Using a previously validated assay (Rice et al., 2013), we recorded an increased expression of ISGs in peripheral blood in five of the seven patients assessed. Importantly, positive serial data were available in four patients, including two patients sampled seven and four times over a period of more than 2 and $6 \mathrm{yr}$, respectively (Table S1, Fig. 2 A, and Fig. S1 D). Although in two probands, ISG expression was not increased on the single occasion tested, we observed a markedly increased level of IFN- $a$ protein in the CSF of one of these individuals. 
Type I IFN signaling in THP-1 cells upon ATAD3A knockdown

Given previous data showing that the two recurrent ATAD3A missense substitutions identified in our patients act as dominantnegative alleles (Cooper et al., 2017; Harel et al., 2016), we performed ATAD3A silencing by lentiviral transduction of THP-1 cells with two different short hairpin RNAs (shRNAs) targeting ATAD3A. $4 \mathrm{~d}$ after transduction, knockdown of ATAD3A mRNA resulted in a decrease in ATAD3A protein (Fig. $2 \mathrm{~B}$ ) and an increase in IFN- $\beta$ and representative ISG expression (IFI27, IFI44L, IFIT1, ISG15, RSAD2, and SIGLEC1; Fig. 2 C). We also observed increased IRF3 phosphorylation, a key event upstream of IFN- $\beta$ induction, and ISG15 protein expression (Fig. 2 F). These data were confirmed by CRISPR editing, leading to downregulation of ATAD3A in THP-1 cell pools using two different guide RNAs, where we also recorded increased phosphorylated IRF3 upon loss of ATAD3A (Fig. S2, A-D).

\section{Definition of the pathway mediating type I IFN signaling upon ATAD3A knockdown}

In mammalian cells, immune responses to viral infection involve host-encoded nucleic acid-binding pattern recognition receptors, including the cytosolic sensors of dsRNA (melanoma differentiation-associated protein 5 [MDA5] and retinoic acidinducible gene I [RIG-I], both signaling through mitochondrialantiviral signaling protein [MAVS]), and DNA, most particularly cyclic GMP-AMP synthase (cGAS), signaling through stimulator of IFN genes (STING; Fig. 2 D). We noticed increased phosphorylation of STING, indicative of STING activation, upon ATAD3A knockdown in WT THP-1 cells (Fig. 2 F). To explore the pathway mediating the observed IFN induction, we compared the results of knockdown of ATAD3A in WT THP-1 cells and characterized THP-1 cells null for cGAS, STING, or MAVS. Increased STING phosphorylation and enhanced IFN signaling, evidenced by IFN- $\beta$ and ISG up-regulation, IRF3 phosphorylation, and ISG15 protein induction, after ATAD3A knockdown by shRNA and CRISPR editing, was preserved in THP-1 cells null for MAVS as in WT cells, but such enhanced signaling was abrogated in the absence of either cGAS or STING (Fig. 2, E and F; and Fig. S2 E).

\section{IFN signaling is dependent on the presence of mtDNA upon ATAD3A knockdown}

Considering the role of ATAD3A in mtDNA maintenance and its interaction with the mtDNA nucleoid organizer TFAM (mitochondrial transcription factor A; He et al., 2012; Zhao et al., 2019), we suspected that cytosolic relocalization of mtDNA, and subsequent sensing by cGAS, might be driving IFN stimulation (West et al., 2015). To explore this further, we analyzed the effect of the knockdown of ATAD3A in THP-1 cells transiently depleted of mtDNA (Fig. 3 A). Compared with UT THP-1 cells, knockdown of ATAD3A in mtDNA-depleted THP-1 cells induced lower levels of expression of IFN- $\beta$ and ISGs (Fig. 3 B), while the response to DNA stimulation remained unchanged (Fig. $3 \mathrm{C}$ ). The induction of IRF3 phosphorylation and ISG15 protein by ATAD3A knockdown was also abrogated by mtDNA depletion (Fig. 3 D). As expected, knockdown of TFAM led to enhanced IFN signaling (considered due to mtDNA cytosolic leakage; West et al., 2015), which was again lost in mtDNA-depleted THP-1 cells (Fig. 3, B and D).
Type I IFN signaling upon mutant ATAD3A overexpression

In trying to assess the functional effect of the p.(Gly355Asp) and p.(Arg528Trp) substitutions in the context of IFN signaling, we observed that overexpression of ATAD3A (WT or mutant) significantly compromised cell survival in THP-1 cells, U2OS cells, and primary healthy fibroblasts (data not shown), consistent with previous reports of defects induced by overexpression of WT AAA ATPases (Evans et al., 2005; Harel et al., 2016). To circumvent this problem, we expressed these mutations in U2OS cells stably expressing STING and endogenous cGAS (Chen et al., 2020) and treated the cells with the caspase inhibitor Q-VD-OPh so as to block caspase-mediated apoptosis (Rongvaux et al., 2014). In this way, we were able to avoid cell death secondary to ATAD3A overexpression and observed increased ISG expression with both mutations (Fig. 3, E and F). Importantly, a similar effect was seen with a dominant-negative Walker A dead (WD) K358A mutation, suggesting a dependency on the ATPbinding function of ATAD3A, and confirming a dominantnegative action of ATAD3A mutations on IFN signaling.

\section{ATAD3A patient-derived cells show enhanced IFN signaling dependent on DNA sensing and the presence of mtDNA}

Our knockdown approaches in THP-1 suggested that loss of ATAD3A function leads to IFN signaling due to DNA sensing of mtDNA. In primary human fibroblasts, ATAD3A knockdown also led to ISG induction (Fig. 4, A and B), dependent on cGAS (Fig. $4 \mathrm{C}$ ). Interestingly, this was independent of TFAM expression (Fig. 4, A and B). To verify these findings in the context of the disease-causing dominant-negative mutations, we assessed IFN signaling in patient fibroblasts and peripheral blood mononuclear cells (PBMCs). ATAD3A and TFAM were expressed at levels similar in patients and controls (Fig. 4 E; Fig. S2, G and $\mathrm{H}$; and Fig. S3, A and B). We observed IFN- $\beta$ and ISG upregulation at the mRNA and protein levels in patient-derived fibroblasts and PBMCs, albeit to different extents between patients (Fig. 4, D and E; and Fig. S2 F). To simplify our readout, we derived an IFN score from the median fold change in expression of six or seven ISGs (Fig. $4 \mathrm{D}$ and Fig. S2 I; details in figure legends). As with ATAD3A knockdown cells, IFN signaling involved cGAS DNA sensing, but not MAVS-dependent RNA sensing (Fig. $4 \mathrm{~F}$ and Fig. S3 D). Total mtDNA levels were not increased in patient fibroblasts compared with controls (Fig. S3 C), ruling out a variation in mtDNA levels as a cause of IFN induction. As with ATAD3A knockdown in THP-1 cells, IFN signaling was abrogated after mtDNA depletion with $2^{\prime}, 3^{\prime}$-dideoxycytidine (ddC) treatment (Fig. 4, G and H; and Fig. S3 F), while the response to DNA stimulation remained unchanged in mtDNA-depleted cells (Fig. S3 E).

\section{ATAD3A dysfunction leads to cytosolic release of mtDNA}

The above data were consistent with IFN signaling due to the cytosolic release of mtDNA sensed by cGAS upon ATAD3A dysfunction. This phenomenon has been reported to depend on mitochondrial outer membrane opening via BAX/BAK1 macropores, VDAC1 channels, or a contribution of the mitochondrial permeability transition pore mPTP (Kim et al., 2019; McArthur et al., 2018; Riley et al., 2018; Yu et al., 2020). Although we did 

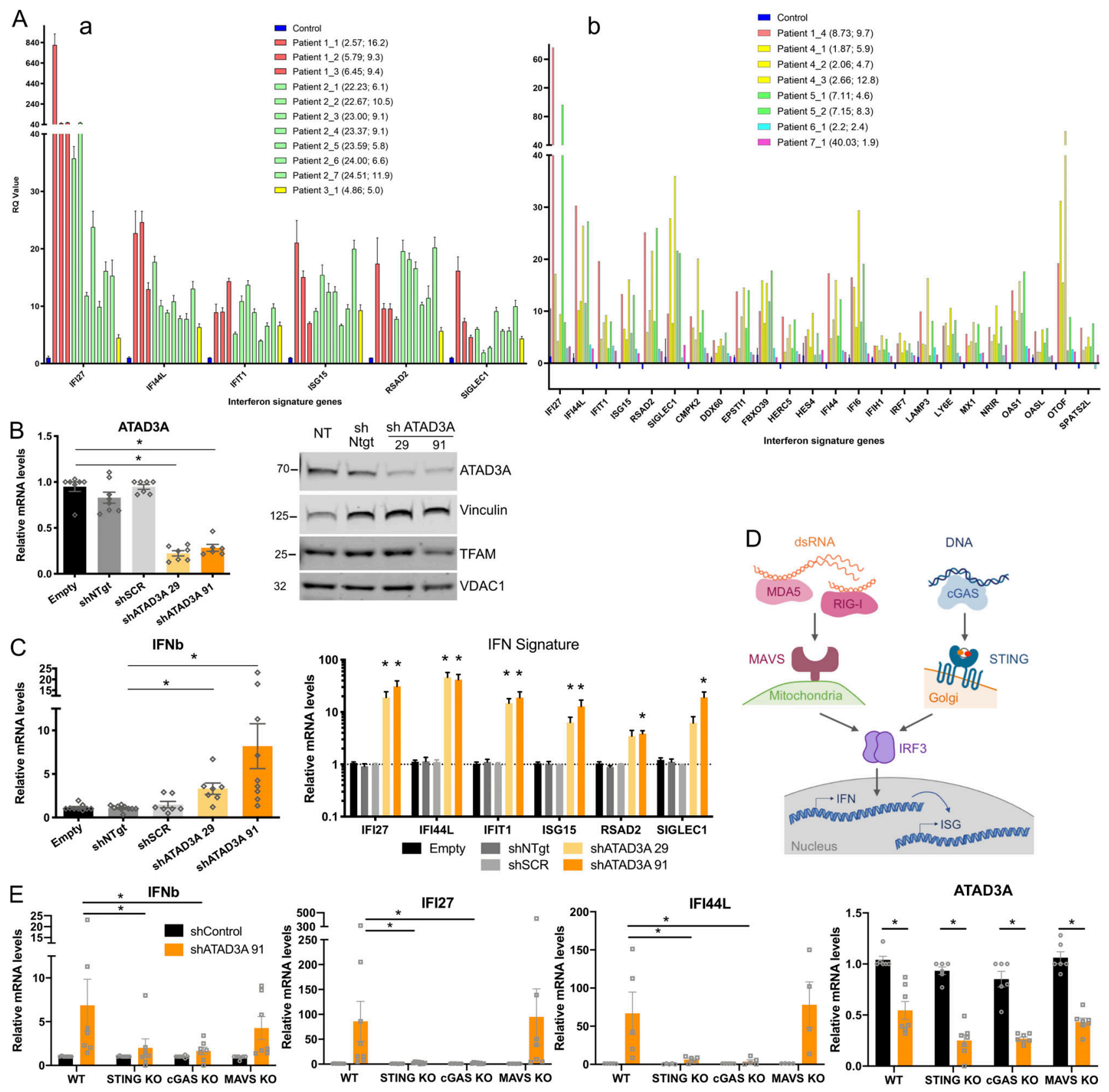

F
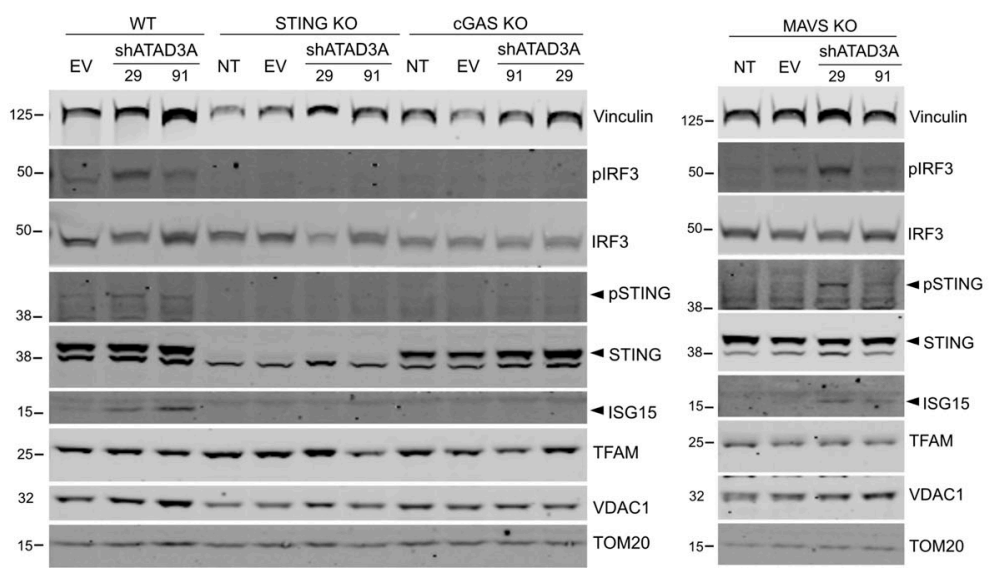

Figure 2. ATAD3A dysfunction leads to up-regulated IFN signaling in patient blood and in THP-1 cells through the cGAS-STING pathway. (A) Enhanced ISG mRNA expression in peripheral whole blood recorded in five of seven patients with ATAD3A mutations. Subpanel a shows the expression of six ISGs 
measured using TaqMan probes, and subpanel b shows a larger panel of 24 genes assessed using a NanoString platform. Colored bars indicate different patients. The first number in the brackets is the decimalized age at the time of sampling and the second the IFN score (calculated according to the median fold change in relative quantification of the ISGs compared with 29 (a) or 27 (b) controls (blue bars; normal < 2.466 [a] or < 2.75 [b]). (B) Knockdown of ATAD3A gene expression (qPCR; left) and of ATAD3A protein (Western blot; right) in THP-1 cells using two shRNAs (shATAD3A 29 and shATAD3A 91) compared with controls (transduction with a vector with no shRNA [Empty], two different unspecific scrambled shRNAs [shNTgt and shSCR]) or non-transduced (NT) and analyzed $4 \mathrm{~d}$ later. For qPCR, mean values of six or seven independent experiments are shown and are expressed as the ratio of the mRNA levels, normalized to housekeeping gene HPRT mRNA, in indicated conditions to that in the empty condition. * indicates statistical significance (P < 0.05$)$ using a Kruskal-Wallis test with Dunn's post hoc. Western blot images are representative of four experiments. VDAC1 and TFAM are mitochondrial proteins, and vinculin is a loading control. (C) Knockdown of ATAD3A leads to increased expression of the IFN- $\beta$ (IFNb) gene and six representative ISGs (IFI27, IFI44L, IFIT1, ISG15, RSAD2, and SIGLEC1) compared with control vectors (Empty, shNTgt, and shSCR) in cells treated as in B. Mean values of 7-10 independent experiments are shown and are expressed as the ratio of the mRNA levels, normalized to housekeeping gene HPRT mRNA, in indicated conditions to that in the empty condition. For IFNb, * indicates significance $(P<0.05)$ in Kruskal-Wallis test with Dunn's post hoc. For ISGs, ${ }^{*}$ indicates statistical significance $(P<0.05)$ in two-way ANOVA with Dunnett's multiple comparison test to shSCR. (D) Schematic of cytosolic nucleic acid sensing pathways; cytosolic dsRNA is sensed by RIG-I like receptors RIG-I and MDA5, triggering MAVS activation situated on mitochondria, and cytosolic DNA is detected by cGAS, signaling through STING on Golgi membranes. Subsequently, upon phosphorylation and dimerization, transcription factor IRF3 induces the expression of IFN- $\beta$ (IFN), leading to the up-regulation of ISG expression. (E) Knockdown of ATAD3A with shATAD3A 91 in WT THP-1 cells and in cells null for either cGAS, STING, or MAVS. The increased expression of IFN- $\beta$ (IFNb) and two representative ISGs (IFI27 and IFI44L) seen in WT THP-1 cells is lost in cells that are null (KO) for either cGAS or STING, while THP-1 cells null for MAVS behave similar to WT cells. Mean values of seven to eight independent experiments are shown and are expressed as the ratio of the mRNA levels, normalized to housekeeping gene HPRT mRNA, in indicated conditions to that with control shRNA ("shControl": shNTgt or shSCR). * indicates statistical significance ( $P$ < 0.05 ) using two-way ANOVA with Dunnett's multiple comparison test. (F) Western blot analysis of ATAD3A knockdown compared with NT cells, or cells treated with an EV, showing an increase in the expression of phosphorylated IRF3, phosphorylated STING, and ISG15 (bottom band) protein in WT and MAVS KO cells, but not in STING KO or cGAS KO cells. One experiment representative of three is shown. TOM20, VDAC1, and TFAM are mitochondrial proteins, and vinculin is a loading control.

not detect any effect of down-regulating BAX and BAK1 or inhibiting their oligomerization with $\mathrm{BAX}$ inhibitor peptide V5 (Fig. S3, H-J), we observed that VDAC1 inhibition with 4,4'diisothiocyanostilbene-2,2' -disulfonic acid (DIDS) led to a partial rescue of IFN- $\beta$ and ISG up-regulation in ATAD3A patient fibroblasts (Fig. 5 A and Fig. S3 G). Notably, while we did not observe any abnormality of mitochondrial network morphology on confocal microscopy in patient fibroblasts (Fig. 5, B and C), down-regulation of ATAD3A in fibroblasts led to the accumulation of cytosolic DNA foci observed by confocal microscopy after staining dsDNA and mitochondria (Fig. 5 D), reminiscent of mtDNA released into the cytosol (McArthur et al., 2018; Yu et al., 2020). Rapamycin is an anti-inflammatory drug that showed a beneficial effect on the features of systemic sclerosis in patients 2 and 3 . As an inhibitor of mTOR, rapamycin is also an inducer of general autophagy, including mitophagy, the removal of damaged mitochondria (Li et al., 2014). Of interest then, we observed a marked decrease of ISG and IFN- $\beta$ expression in patient fibroblasts following rapamycin treatment (Fig. 5 A and Fig. S3 G).

We report mutations in ATAD3A to be associated with an upregulation of type I IFN signaling equivalent to that seen in established type I interferonopathies (Fig. S1 D and Fig. S2 I). The identification of asymptomatic individuals heterozygous for ATAD3A null alleles (Harel et al., 2016), and the results of in vitro functional testing relating to pathogenic missense mutations, including the substitutions recorded in our patients (Cooper et al., 2017; Harel et al., 2016, this work), indicate that such missense mutations act in a dominant-negative fashion, likely through interfering with normal oligomer function (Gilquin et al., 2010). Although we cannot rule out the possibility of some degree of IFN signaling mediated by RNA, knockdown of ATAD3A in THP-1 cells induced type I IFN signaling that was dependent on the cGAS-STING dsDNA-sensing pathway. Concordantly, removal of mtDNA abrogated this effect. Both findings were also observed in patient fibroblasts. Mitochondrial dysfunction and altered mitochondrial ultrastructure have been reported to cause mtDNA leakage into the cytosol (Kim et al., 2019). Thus, one mechanism to explain the observed cGASSTING-mediated induction of IFN would be through cytoplasm sensing of mtDNA. In mammalian cells, ATAD3A is a mitochondrial membrane protein enriched at contact sites between mitochondria and the endoplasmic reticulum (Baudier, 2018). We did not observe significant changes in mitochondrial protein (VDAC1 and TOM20) levels in THP-1 cells or patient-derived fibroblasts (Fig. 2 B, Fig. 3 D, Fig. 4 E, Fig. S2 D, and Fig. S3 B), suggesting that mitochondrial mass per se is unaffected by ATAD3A dysfunction. Cooper et al. recorded fragmented mitochondria on overexpression of the p.(Gly355Asp) mutation in fibroblasts and neurons derived from patient fibroblasts with this substitution (Cooper et al., 2017). However, in keeping with a previous study (Dorison et al., 2020), we did not observe any abnormalities in fibroblasts from patients 2-4 on quantification of mitochondrial network shape using a supervised machine learning approach (Fig. 5, B and C) or in ATAD3A-silenced fibroblasts (Fig. 5 D). Aggregated/larger mtDNA foci have been described in cells of patients with ATAD3A deletions (Desai et al., 2017). Down-regulation of TFAM, the mtDNA nucleoid organizer that ATAD3A binds (He et al., 2012), also causes enlarged mtDNA foci and leads to cytosolic leakage of mtDNA (West et al., 2015). We found total TFAM protein levels to be similar in patient and control cells (Fig. 4 E), ruling out TFAM loss as a potential mechanism of increased mtDNA sensing. However, mutated ATAD3A could alter the retention of mtDNA by TFAM, as reported when ATAD3A expression and oligomerization are altered (Zhao et al., 2019). Consistent with this, we observed increased numbers of cytosolic DNA foci upon ATAD3A downregulation and a contribution of VDAC1 channels to enhanced IFN signaling, which is worthy of more detailed study. Complementarily to its action as an anti-inflammatory drug (Wang et al., 2021), the effect of rapamycin in patient fibroblasts 

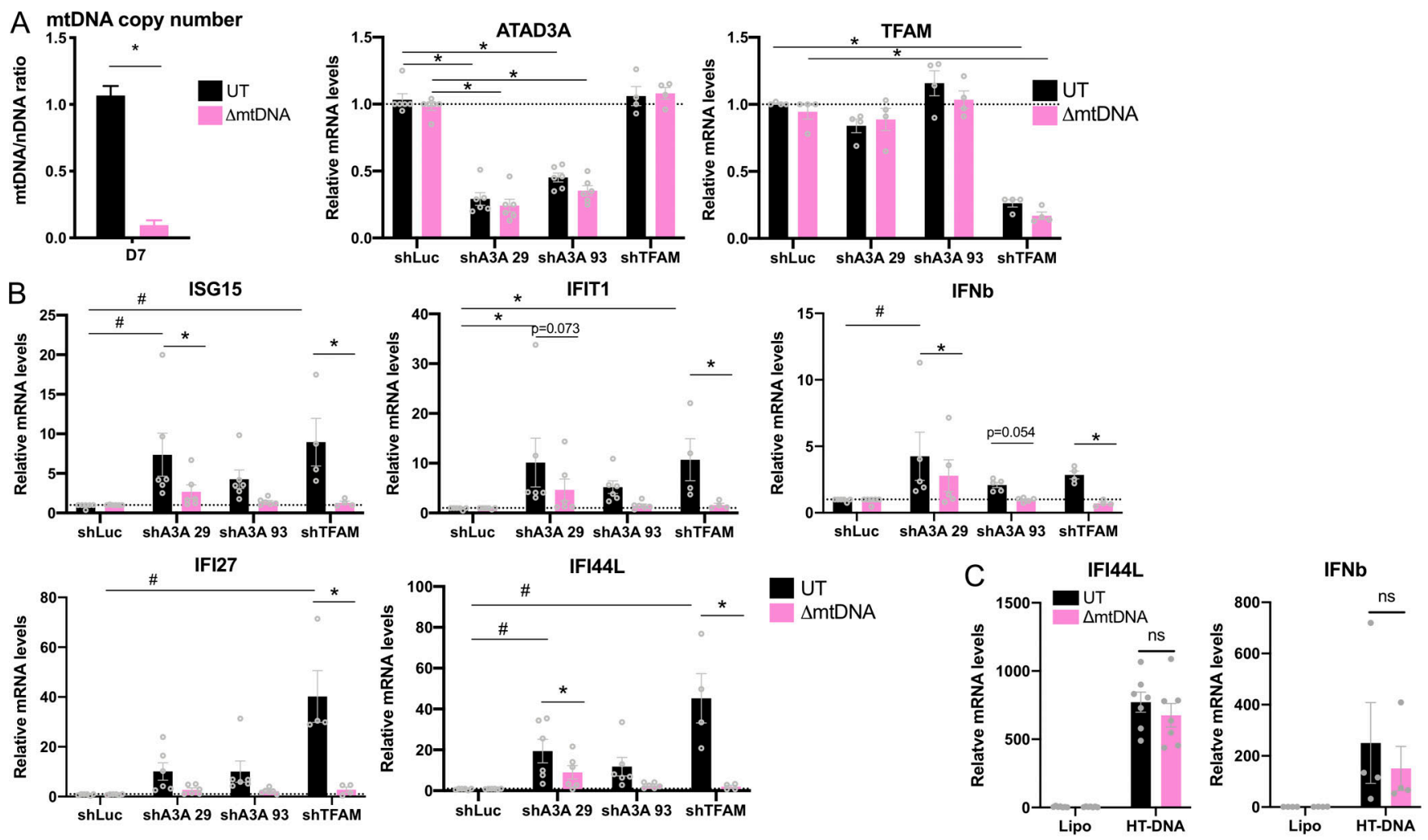

D
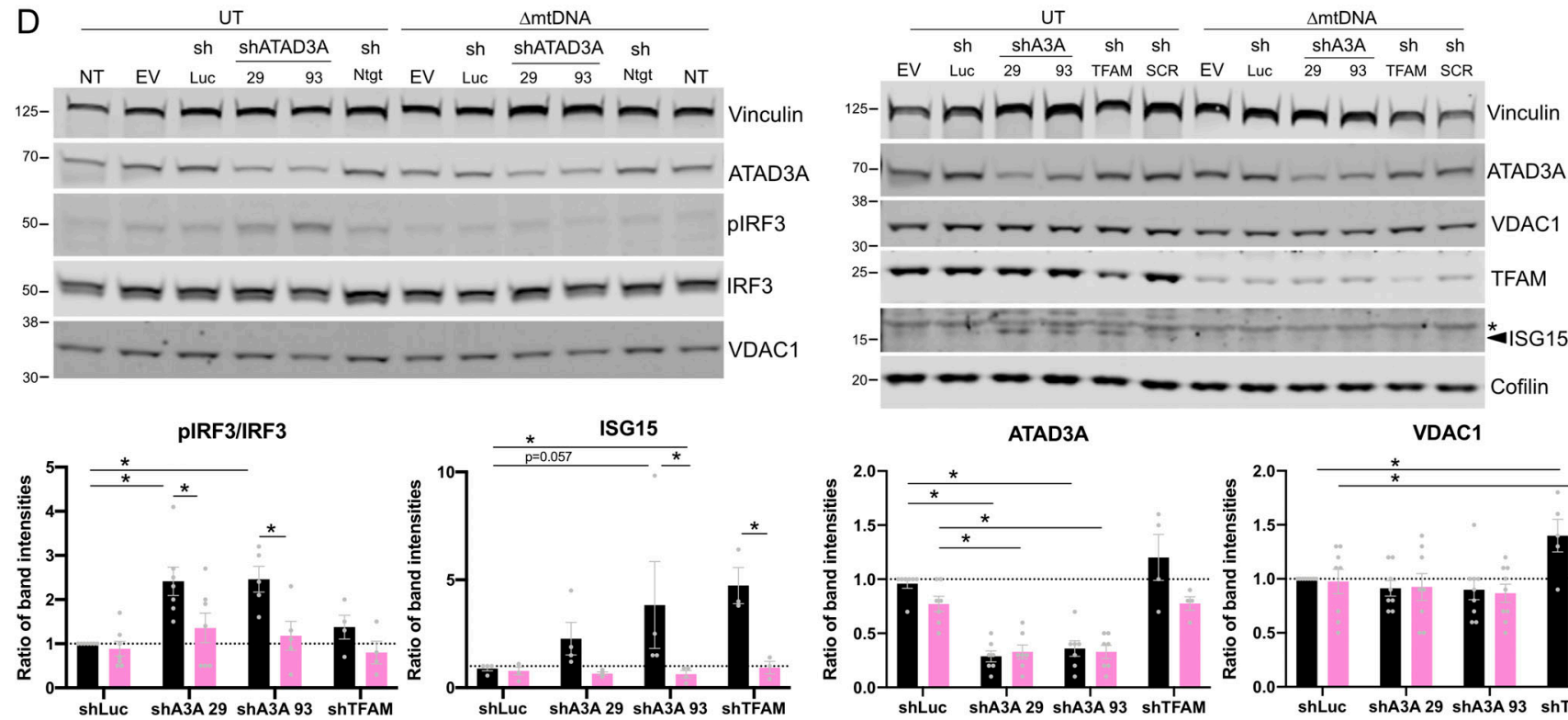

ATAD3A

VDAC1
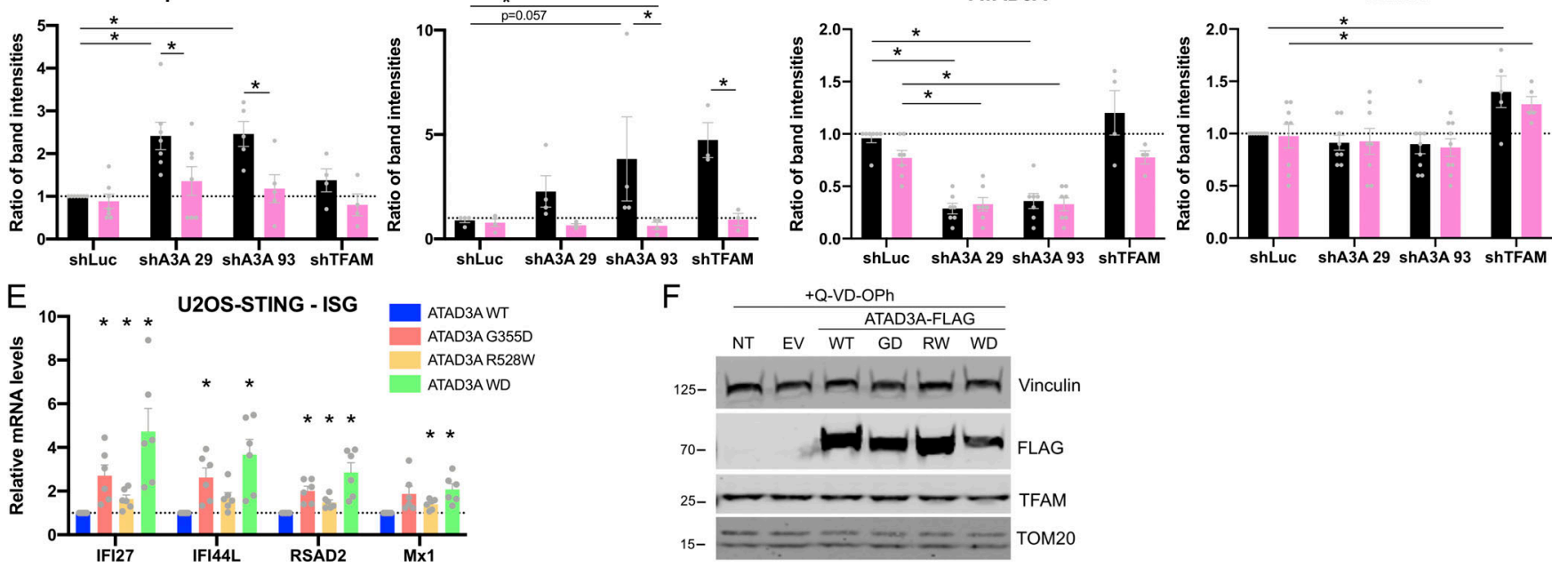

Figure 3. IFN signaling upon ATAD3A silencing in THP-1 cells is dependent on the presence of mtDNA. (A) Left: Depletion of mtDNA in THP-1 cells. Graph shows qPCR analysis of mtDNA copy number per cell, expressed as the ratio of the DNA quantity of the mitochondrial gene (MT-COXII) over the nuclear gene (GAPDH), in total DNA isolated from UT THP-1 cells or cells treated for $7 \mathrm{~d}$ with low-dose ethidium bromide to deplete mtDNA ( $\triangle \mathrm{mtDNA}$ ). The average of four independent experiments is shown. ${ }^{*}$ indicates statistical significance $(P<0.05)$ using a Mann-Whitney test. Right: Knockdown of ATAD3A or TFAM 
assessed by qPCR using either of two shRNAs targeting ATAD3A (shA3A 29 and shA3A 93), or an shRNA targeting TFAM (shTFAM) in mtDNA-depleted THP-1 cells compared with control shRNA (shLuc). * indicates statistical significance $(P<0.05)$ in two-way ANOVA with Dunnett's multiple comparison test compared with respective shLuc. (B) Upon knockdown of either ATAD3A or TFAM, the increase in the expression of IFN- $\beta$ (IFNb) and of four representative ISGs (ISG15, IFIT1, IFI27, and IFI44L) seen in UT THP-1 cells was lost when THP-1 cells depleted of mtDNA were used. Mean values of five to six independent experiments are shown and are expressed as the ratio of the mRNA levels, normalized to housekeeping gene HPRT mRNA, in indicated conditions to that in the control shLuc condition. \# indicates statistical significance $(P<0.05)$ in two-way ANOVA with Dunnett's multiple comparison test compared with respective shLuc, and * indicates statistical significance in two-way ANOVA with Sidak's multiple comparison test comparing $\triangle m$ mDNA and UT conditions for each shRNA. (C) qPCR analysis of IFI44L and IFNb expression in UT or $\triangle \mathrm{mtDNA}$ THP-1 cells after a 24-h stimulation with $1 \mu \mathrm{g} / \mathrm{ml}$ HT-DNA. Mean values of three to four independent experiments are shown. ns indicates non-significance in two-way ANOVA with Sidak's multiple comparison test. (D) Western blot analysis of ATAD3A and TFAM knockdowns described in A compared with NT cells or cells treated with an EV or a control shRNA (shNTgt/shLuc), showing an increase in the expression of phosphorylated IRF3 (left) and ISG15 (right, bottom band; ${ }^{*}$, nonspecific band) proteins, abrogated by mtDNA depletion. Two representative experiments are shown. Quantification of band intensities is expressed as the ratio of phosphorylated IRF3/total IRF3 or the ratio of ISG15, ATAD3A, and VDAC1 (mitochondrial protein control) protein levels normalized to vinculin, averaged from four to seven independent experiments. ${ }^{*}$ indicates statistical significance $(P<0.05)$ in two-way ANOVA with Sidak's multiple comparison test comparing relevant shRNA to control shLuc in each cell line and comparing UT and mtDNA-depleted cells for each shRNA. (E) mRNA expression analysis assessed by qPCR of ISGs IFI27, IFI44L, RSAD2, and Mx1 in U2OS cells stably expressing STING (U2OS-STING), transfected with FLAG-tagged ATAD3A WT or mutant G355D, R528W, and WD plasmids, treated with $10 \mu$ M QVD-OPh after 24 h and harvested $24 \mathrm{~h}$ later. Expression levels are expressed as the fold of levels in WT transfected cells. Mean values of six independent experiments, statistically analyzed using two-way ANOVA and Dunnett's multiple comparisons test ( $\left.{ }^{*}, \mathrm{P}<0.05\right)$. (F) Western blot analysis of FLAG, vinculin, TFAM, and TOM20 in whole-cell lysates of U2OS-STING treated and harvested as in E (GD, G355D; RW, R528W). Representative results from four independent experiments.

suggests that it might act on a cell-intrinsic basis, possibly inducing the autophagy of cytosolic DNA or of damaged mitochondria leaking mtDNA, to curb mtDNA detection and IFN signaling. Of note, the mitophagy-inducing potential of rapamycin has been implicated in therapeutic strategies relating to mitochondrial disease (Li et al., 2014; Villanueva Paz et al., 2016).

Two of the patients that we ascertained demonstrate signs consistent with standardized criteria for a diagnosis of the autoimmune disorder systemic sclerosis (van den Hoogen et al., 2013), with classical histological features of systemic sclerosis (i.e., fibrosis, loss of skin adnexae, and deep lymphocytic perivascular infiltrates) on skin biopsy in both cases. The positivity for multiple autoantibodies, and elevated levels of gammaglobulin observed in patient 2, are suggestive of an autoimmune phenomenon, although these features were not present in patient 3 . We note the frequent presence of autoantibodies in the type I interferonopathy COPA syndrome (Lepelley et al., 2020). The pathology of systemic sclerosis remains unclear, with the phenotype likely representing the end point of a heterogeneous set of disease processes (Bhattacharyya et al., 2011). Genetic association (López-Isac et al., 2019), gene expression (Brkic et al., 2016), and experimental studies (Ah Kioon et al., 2018; Wu et al., 2019) indicate a link between systemic sclerosis and up-regulated type I IFN signaling, presumed to be induced by self-derived DNA or RNA (Barrat et al., 2016). The source of such nucleic acids remains unclear. While the potential of mtDNA to induce cytoplasmic cGAS-STING-mediated IFN signaling (West et al., 2015; West and Shadel, 2017), and thereby promote autoimmunity (Kim et al., 2019), has been recently highlighted (Crow, 2019), it is possible that other inflammatory signaling pathways might also be relevant (Zhou et al., 2011; Yu et al., 2020).

Although mutations in ATAD3A are associated with a disturbance of mitochondrial structure, oxidative phosphorylation enzyme deficiency has been only variably recorded in affected individuals (Desai et al., 2017; Gunning et al., 2020; Harel et al., 2016; Peralta et al., 2019; Frazier et al., 2021), indicating the possibility of disease-related pathology due to other than a failure of energy production. While the recurrent observation of absence of the eyelashes and eyebrows, seen in four of seven patients in our series, suggests a developmental aspect to the function of ATAD3A, our findings indicate that type I IFN induction might also represent a pathogenic factor. In this regard, we note that intracranial calcification, a sign observed in a number of type I interferonopathies, was present in patient 6 and that dystonia, peripheral neuropathy and hypertrophic cardiomyopathy, as recorded in some patients with mutations in ATAD3A, are (also) features of IFN-related disease (Crow et al., 2015). Additionally, isolated spastic paraparesis (as seen in patient 7) is a well-recognized manifestation of mutations in several type I interferonopathy-related genes (Crow et al., 2014).

Our clinical data are important in extending the phenotype associated with mutations in ATAD3A to include an association with systemic sclerosis. Further, we note the marked difference in phenotype between the mother (patient 7 here; lower limb spastic paraparesis with normal intellect) and her son (four-limb spastic dyskinesia and severe intellectual delay) as reported by Cooper et al. (2017). Variability in clinical expression, including purely neurological disease (Livingston et al., 2014), frank autoimmunity (Van Eyck et al., 2015), and complete clinical nonpenetrance (Lepelley et al., 2020; Rice et al., 2020), is highly characteristic of the type I interferonopathies.

To our knowledge, mutations in ATAD3A represent the first example of a Mendelian mitochondrial disease directly implicating mtDNA in type I IFN induction. The relative contribution of enhanced type I IFN signaling to the ATAD3A-associated phenotype is yet to be determined; however, the advent of strategies to block IFN signaling may allow this question to be tested in an experimental medicine setting in the near future.

\section{Materials and methods}

\section{Samples obtained from patients}

Samples were obtained from the probands and parents with written informed consent. The study was approved by the Comité de Protection des Personnes (ID-RCB/EUDRACT: 2014A01017-40) and the Leeds (East) Research Ethics Committee (10/ H1307/132). 


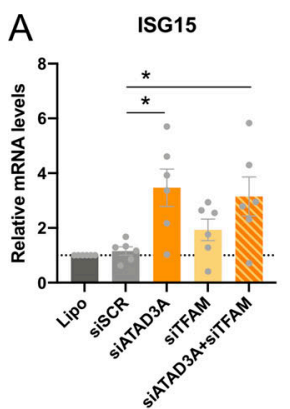

B

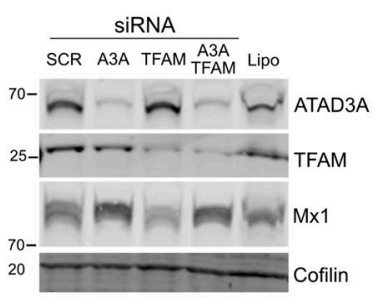

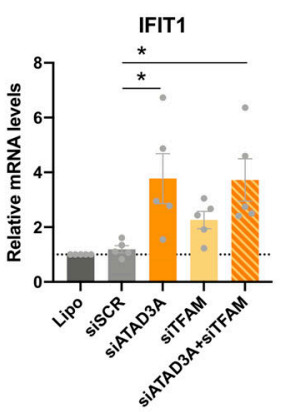

C ISG15

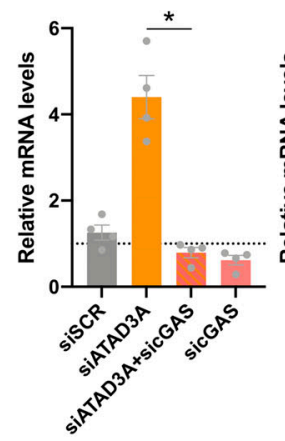

IFN Signature
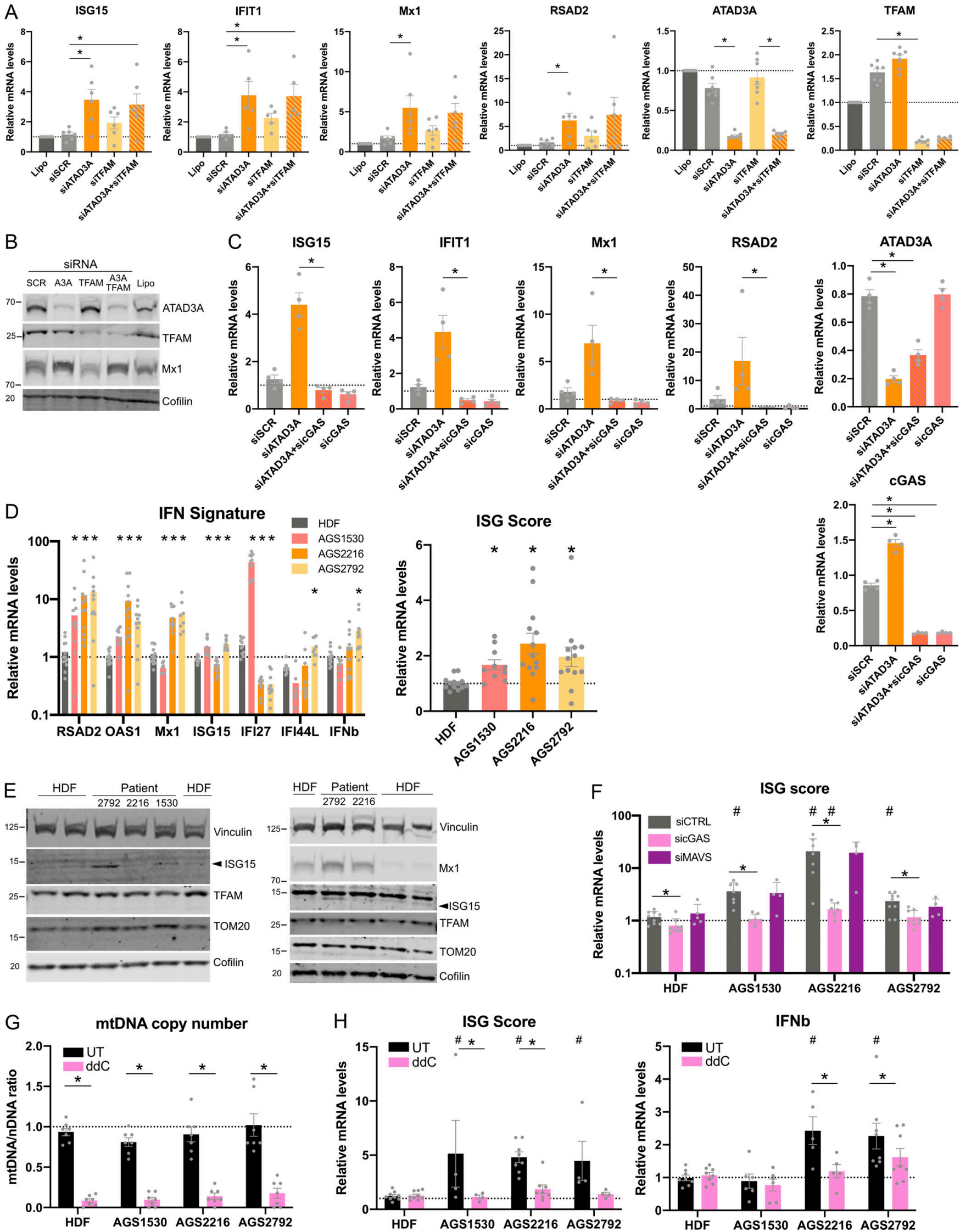

Figure 4. IFN signaling in fibroblasts from ATAD3A patients is CGAS and mtDNA dependent. (A) ISGs, ATAD3A, and TFAM mRNA expression in control primary fibroblasts after down-regulation of ATAD3A (siATAD3A), TFAM (siTFAM), or both (siATAD3A+siTFAM) by siRNA, treatment with control siRNA (siSCR) 

to that in siSCR. Mean values and data points of six to seven independent experiments are shown. ${ }^{*}$ indicates statistical significance $(P<0.05)$ in Friedman test with Dunn's multiple comparison test to the siSCR condition. (B) Protein expression in primary fibroblasts treated as in A of a representative ISG Mx1, ATAD3A, and TFAM assessed by Western blot. Cofilin is a loading control. (C) ISGs, ATAD3A, and cGAS mRNA expression in control primary fibroblasts after downregulation of ATAD3A (siATAD3A), cGAS (sicGAS), or both (siATAD3A+sicGAS) by siRNA, treatment with control siRNA (siSCR) or transfection reagent alone (Lipo) for $72 \mathrm{~h}$, expressed as in A. Mean values and data points of six to seven independent experiments are shown; three datasets are also displayed in A. * indicates statistical significance $(P<0.05)$ in Mann-Whitney test between siATAD3A and siATAD3A+sicGAS conditions for ISGs, or statistical significance ( $<$ < 0.05 ) in one-way ANOVA for ATAD3A and cGAS. (D) Left: ISG and IFN- $\beta$ (IFNb) mRNA expression in primary fibroblasts from control human dermal fibroblasts (HDF; average of three) or from ATAD3A patients 2, 3, and 4 (AGS1530, AGS2216, and AGS2792, respectively), expressed as the ratio of the mRNA levels, normalized to housekeeping gene HPRT mRNA, in indicated conditions to that in one control HDF. Mean values and data points of 10-13 independent experiments are shown. * indicates statistical significance $(P<0.05)$ in two-way ANOVA with Dunnett's multiple comparison test. Right: ISG mRNA expression represented as an ISG score (i.e., the median fold change of mRNA levels of six ISGs [RSAD2, OAS1, Mx1, IFI27, ISG15, and IFI44L]) in control and ATAD3A patient-derived primary fibroblasts shown on the left. ${ }^{*}$ indicates statistical significance $(P<0.05)$ in Kruskal-Wallis test with Dunn's multiple comparison. (E) Protein expression in patient-derived primary fibroblasts of two representative ISGs (ISG15 and Mx1) assessed by Western blot. TFAM and TOM20 are mitochondrial proteins, and cofilin and vinculin are loading controls. Arrowhead indicates relevant band for ISG15. (F) ISG mRNA expression, represented as an ISG score (i.e., the median fold change of mRNA levels of seven ISGs [RSAD2, OAS1, Mx1, IFI27, ISG15, IRF7, and IFI44L] expressed as in D) in control and ATAD3A patient-derived primary fibroblasts, after down-regulation of cGAS (sicGAS) or MAVS (siMAVS) by siRNA or treatment with control siRNA (siCTRL). Mean values and data points of six to nine independent experiments are shown. ${ }^{*}$ indicates statistical significance (P < 0.05$)$ in two-way ANOVA with Dunnett's multiple comparison tests between siRNA conditions for each cell line, and \# indicates statistical significance (P < 0.05) in two-way ANOVA with Dunnett's multiple comparison test between control and patient-derived fibroblasts for each siRNA condition. (G) Depletion of mtDNA in patient-derived primary fibroblasts. Graph shows qPCR analysis of mtDNA copy number per cell, expressed as the ratio of the DNA quantity of the mitochondrial gene (MT-COXII) over the nuclear gene (GAPDH), in total DNA isolated from UT fibroblasts or cells treated for 7-14 d with $100 \mu \mathrm{M}$ ddC to deplete mtDNA. The mean of seven independent experiments is shown. * indicates statistical significance $(P<0.05)$ in two-way ANOVA with Sidak's multiple comparison test comparing ddC and UT conditions for each fibroblast line. (H) ISG mRNA expression, represented as an ISG score (i.e., the median fold change of mRNA levels of seven ISGs [RSAD2, OAS1, Mx1, IFI27, ISG15, IRF7, and IFI44L] expressed as in D) and IFN- $\beta$ (IFNb) mRNA expression in control and ATAD3A patient-derived primary fibroblasts, UT or mtDNA-depleted by ddC as in G. Mean values and data points of three to eight independent experiments are shown. ${ }^{*}$ indicates statistical significance (P < $0.05)$ in two-way ANOVA with Sidak's multiple comparison test between UT and ddC conditions. \# indicates statistical significance ( $P$ < 0.05$)$ in two-way ANOVA with Dunnett's multiple comparison test between control and patient-derived fibroblasts for each treatment.

\section{Histological analyses}

Formalin-fixed paraffin-embedded skin biopsy slides were analyzed after H\&E staining.

\section{Genetic studies}

DNA was extracted from whole blood using standard methods. Exome sequencing was performed on genomic DNA from patients 1 and 2 and their parents using a SureSelect Human All Exon kit (Agilent Technologies) for targeted enrichment, and Illumina HiSeq2000 for sequencing. Variants were assessed using the in silico programs SIFT (http://sift.jcvi.org) and Polyphen2 (http://genetics.bwh.harvard.edu/pph2/). Population allele frequencies were obtained from the gnomAD database (http://gnomad.broadinstitute.org). Sanger sequencing was performed to confirm the identified ATAD3A variants. The reference sequence used for primer design and nucleotide numbering was NM_001170535.

\section{IFN status}

Whole blood was collected into PAXgene tubes (Qiagen) and total RNA extracted using a PreAnalytix RNA isolation kit. IFN scores were generated in one of two ways as previously described using TaqMan probes to measure the mRNA expression of six ISGs (IFI27, IFI44L, IFIT1, ISG15, RSAD2, and SIGLEC1) normalized to the expression level of HPRT1 and 18S rRNA (Rice et al., 2013). The median fold change of the ISGs is compared with the median of 29 healthy controls to create an IFN score for each individual, with an abnormal score being defined as greater than 2.46. For NanoString ISG analysis, total RNA was similarly extracted from whole blood with a PAXgene (PreAnalytix) RNA isolation kit. Analysis of 24 genes and 3 housekeeping genes (probes of interest [ $n=24]$ : IFI27, IFI44L, IFIT1, ISG15, RSAD2, SIGLEC1, CMPK2, DDX60, EPSTI1, FBXO39, HERC5, HES4, IFI44, IFI6, IFIH1, IRF7, LAMP3, LY6E, MX1, NRIR, OAS1, OASL, OTOF, and SPATS2L; reference probes [ $n=3]$ : NRDC, OTUD5, and TUBB) was conducted using the NanoString customer designed CodeSets according to the manufacturer's recommendations (NanoString Technologies). Agilent Tapestation was used to assess the quality of the RNA. 100 ng total RNA was loaded for each sample. Data were processed with nSolver software (NanoString Technologies). The data were normalized relative to the internal positive and negative calibrators, the three reference probes, and the control samples. The median of the 24 probes for each of 27 healthy control samples was calculated. The mean NanoString score of the 27 healthy controls +2 SD of the mean was calculated. Scores above this value (2.75) were designated as positive.

Plasma and CSF IFN- $\alpha$ concentrations $(\mathrm{fg} / \mathrm{ml})$ were determined by single-molecule array (Simoa) using a commercial kit for IFN-a2 quantification (Quanterix). The assay was based on a three-step protocol on an SR-X analyzer (Quanterix).

\section{Cell culture}

Human embryonic kidney 293FT cells (catalog no. R70007; Invitrogen) were grown in DMEM (GIBCO) supplemented with $10 \%$ (vol/vol) fetal bovine serum (GIBCO). Primary fibroblasts (three from controls, from patients 2 [AGS1530], 3 [AGS2216], and 4 [AGS2792], and from AGS8/9 patients described in Uggenti et al., 2020) and BJ-5ta fibroblasts (catalog no. CRL-4001; ATCC) were grown in DMEM supplemented with 10\% (vol/vol) fetal bovine serum, with $1 \%$ penicillin-streptomycin and $0.25 \mu \mathrm{g} / \mathrm{ml}$ amphotericin B (GIBCO) added for primary fibroblasts. THP-1 cells were from ATCC (TIB-202) and WT, STING KO, and cGAS 


\section{מ JEM}

A

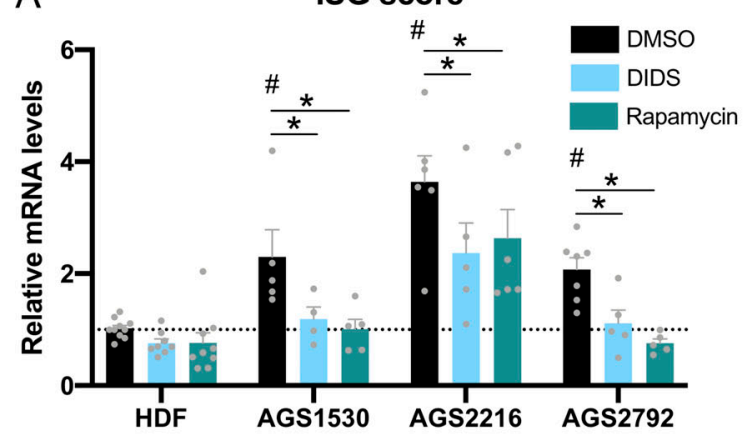

B
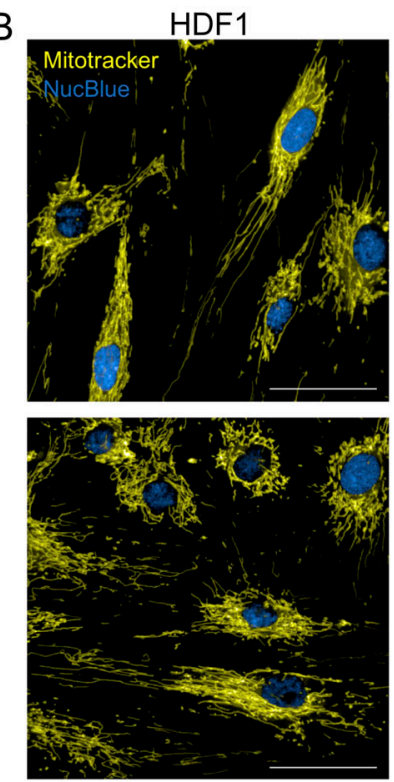

AGS1530

D DNA Mito siLuc
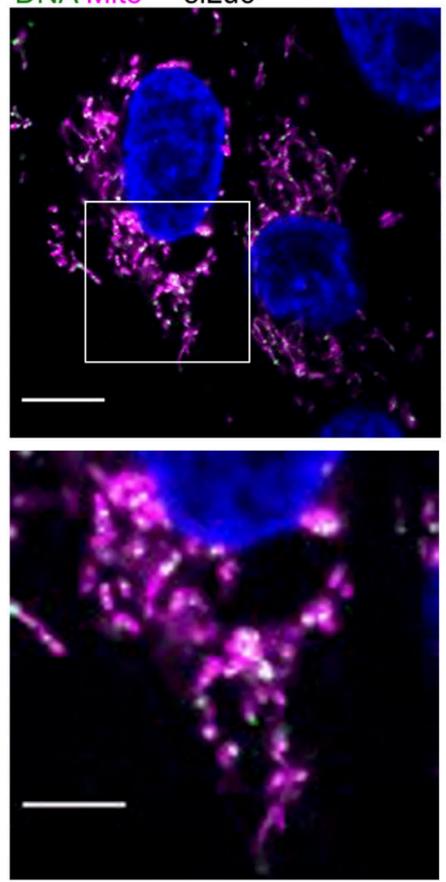
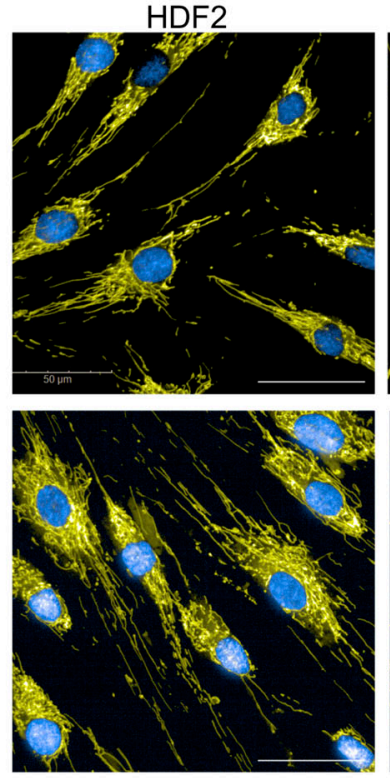

AGS2216

SIATAD3A 1
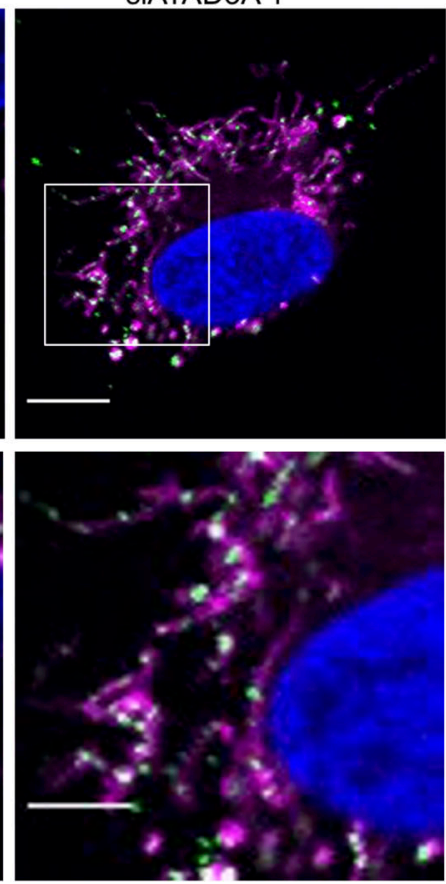
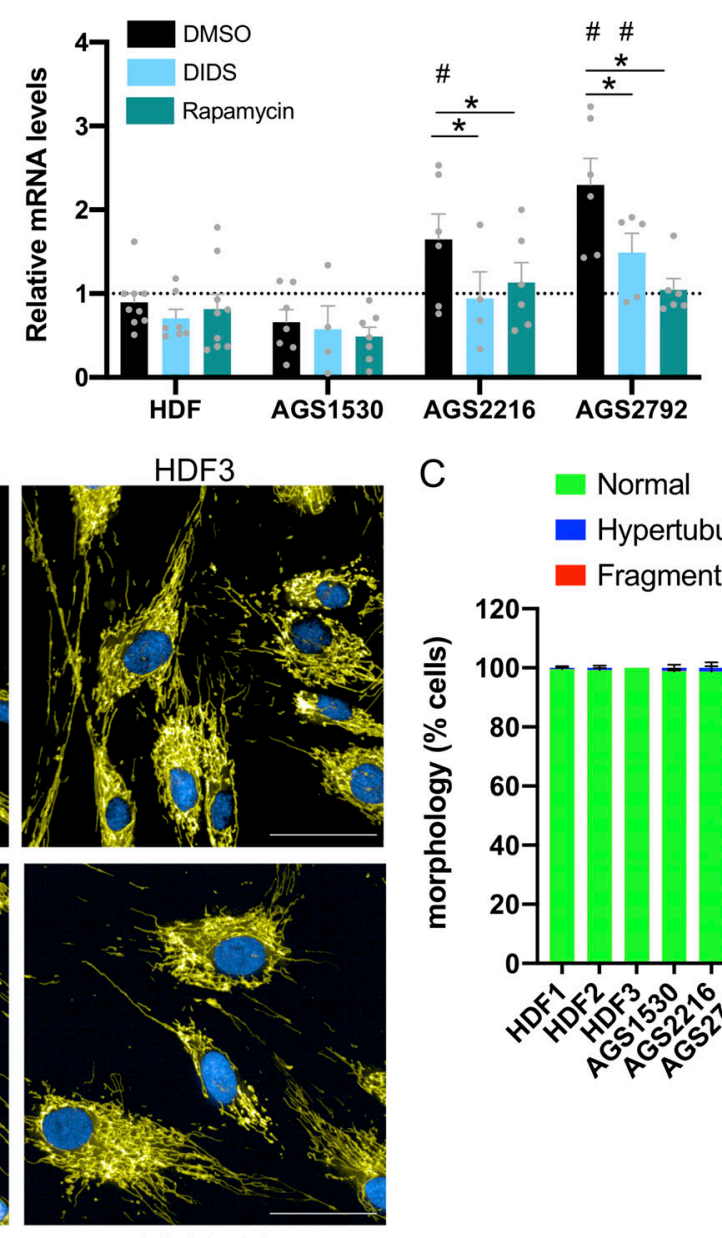

AGS2792
C Normal

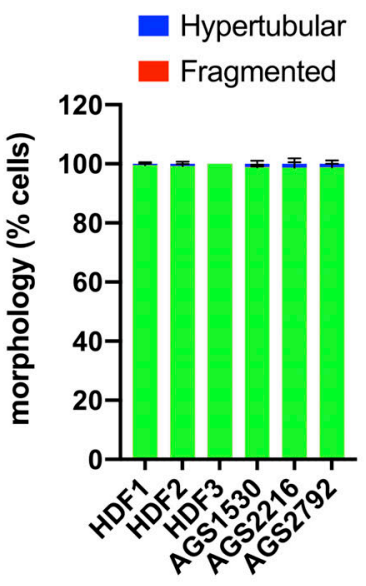

SIATAD3A 2
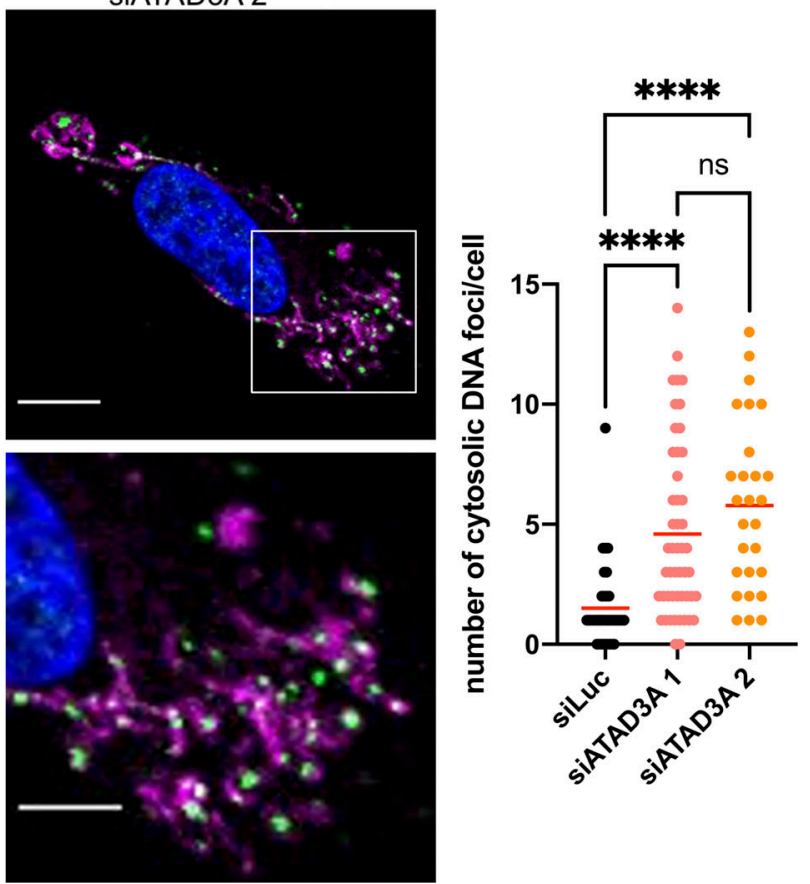

Figure 5. ATAD3A down-regulation in fibroblasts leads to cytosolic release of mtDNA. (A) ISG mRNA expression, represented as an ISG score (i.e., the median fold change of mRNA levels of seven ISGs [RSAD2, OAS1, Mx1, IFI27, ISG15, IRF7, and IFI44L] and IFN- $\beta$ (IFNb) mRNA expression in control and ATAD3A 
patient-derived primary fibroblasts, vehicle-treated (DMSO) or treated with $300 \mu \mathrm{M}$ DIDS or 100 nM rapamycin for $72 \mathrm{~h}$. Mean values and data points of five to seven independent experiments are shown. ${ }^{*}$ indicates statistical significance $(P<0.05)$ in two-way ANOVA with Dunnett's multiple comparison tests between treatment conditions. \# indicates statistical significance $(P<0.05)$ in two-way ANOVA with Dunnett's multiple comparison test between control and patientderived fibroblasts for each treatment. (B) Representative confocal images of control (HDF 1, 2, and 3) and ATAD3A patient-derived primary fibroblasts (AGS1530, AGS2216, and AGS2792) stained for the nucleus (NucBlue in blue) and mitochondria (MitoTracker in yellow). Scale bars, 50 mm. (C) Mitochondrial morphology quantification of control and ATAD3A fibroblasts was performed via supervised machine learning training using HDF 1 cells with fragmented (CCCP-treated), normal (DMSO-treated), and hypertubular (CHX-treated) mitochondria, and automatic single-cell trinary classification. Data represent mean \pm SD of three independent experiments (200-442 cells per cell line), nonsignificant in one-way ANOVA. (D) Representative images of immunofluorescence staining of mitochondria (MitoTracker, magenta), dsDNA (DNA, green), and nucleus (DAPI, blue) in BJ-5ta fibroblasts knocked down for ATAD3A by two siRNAs (siATAD3A 1 and 2), observed by confocal microscopy. Mitochondria/DNA overlap is represented in white. Bottom panel shows a magnification of indicated frames in top panel. Scale bars represent $10 \mu \mathrm{m}$ (top panels) and $5 \mu \mathrm{m}$ (bottom panels). Graph on the right shows a quantification of DNA spots not colocalized with mitochondrial staining per cell. Means are symbolized by red bars. Results averaged from at least 25 cells counted from one experiment representative of three independent experiments. ${ }^{* * *}$, statistical significance $(P<0.0001)$ in one-way ANOVA with Tukey's multiple comparison test.

KO THP-1 cells were from InvivoGen (THP-1 Dual, ref. thpdkostg and thpd-kocgas). MAVS KO THP-1 cell lines were recently generated in the Rehwinkel laboratory (Medical Research Council Weatherall Institute of Molecular Medicine, Oxford, UK; Hertzog et al., 2021 Preprint). All THP-1 cell lines were maintained in RPMI (Invitrogen) supplemented with 10\% (vol/vol) fetal bovine serum, $10 \mathrm{mM}$ Hepes and $0.05 \mathrm{mM}$ 2-mercaptoethanol (GIBCO). U2OS cells (catalog no. HTB-96; ATCC) were maintained in McCoy medium (Invitrogen) supplemented with $10 \%$ (vol/vol) fetal bovine serum. All cell lines were cultured at $37^{\circ} \mathrm{C}$ in $5 \% \mathrm{CO}_{2}$. Primary fibroblasts were treated for $72 \mathrm{~h}$ with vehicle (DMSO; Sigma-Aldrich), $300 \mu \mathrm{M}$ DIDS (309795; Sigma-Aldrich), $100 \mathrm{nM}$ rapamycin (S1039; Selleckchem), or 50-200 $\mu \mathrm{M}$ BAX inhibitor peptide V5 (HY-P0081; MedChemExpress). THP-1 cells and fibroblasts were stimulated for $24 \mathrm{~h}$ with $1 \mu \mathrm{g} / \mathrm{ml}$ herring testes (HT)-DNA (D6898; Sigma-Aldrich) complexed with Lipofectamine 2000 (Invitrogen), according to the manufacturer's instructions, or lipofectamine alone. PBMCs were isolated by Ficoll-Paque density gradient (Lymphoprep; Proteogenix) from the blood of patients and healthy donors. Control PBMCs from healthy donors were obtained from the Etablissement Français du Sang blood bank. Cells were pelleted and kept at $-80^{\circ} \mathrm{C}$ until further analysis.

\section{Knockdown of gene expression by shRNA}

Lentiviral constructs containing shRNA targeting ATAD3A (TRCN0000136391: shATAD3A 91, TRCN0000135329: shATAD3A 29, and TRCN0000136793: shATAD3A 93), TFAM (TRCN0000329820: shTFAM), and control Luciferase (shLuc, SHC007) were made by ligating annealed oligonucleotides into pLKO.1 (TRC cloning vector, a gift from David Root, Broad Institute of MIT and Harvard, Cambridge, MA [plasmid \#10878; Addgene]), according to the RNAi consortium protocol (https://portals.broadinstitute.org/ gpp/public/). pLKO.1 empty vector (Empty/EV) was from Open Biosystems, and pLKO.1 control shRNAs (scramble shSCR, SHC002, and non-target shNTgt, SHC016) were from SigmaAldrich. Lentiviral vectors carrying these constructs were produced by calcium phosphate transfection of 293FT cells with shRNA constructs in combination with packaging vectors psPAX2, a gift from Didier Trono (École polytechnique fédérale de Lausanne, Lausanne, Switzerland; plasmid \#12260; Addgene), and envelope pCMV-VSV-G (plasmid \#8454; Addgene). Medium of $70 \%$ confluent $293 \mathrm{FT}$ in $75-\mathrm{cm}^{2}$ flasks was changed $2 \mathrm{~h}$ before transfection. Calcium phosphate precipitates were prepared by mixing $12.5 \mu \mathrm{g}$ shRNA vectors with $12.5 \mu \mathrm{g}$ psPAX2 and $5 \mu \mathrm{g}$
pCMV-VSV-G in water for a final volume of $875 \mu$ l. $125 \mu \mathrm{l} 2 \mathrm{M}$ $\mathrm{CaCl}_{2}$ and $1 \mathrm{ml} \mathrm{HBS} 2 \mathrm{X}$ (50 mM Hepes, $10 \mathrm{mM} \mathrm{KCl,} 280 \mathrm{mM} \mathrm{NaCl}$, and $1.5 \mathrm{mM} \mathrm{Na}_{2} \mathrm{HPO}_{4}$, pH 7.05) were sequentially added dropwise in slowly vortexed solution. Solutions were incubated at room temperature for 20 min and mixed gently with 293FT supernatant. Medium was replaced by $7 \mathrm{ml}$ culture medium $24 \mathrm{~h}$ later. After 24 more hours, supernatants were collected, centrifuged at $1,700 \mathrm{rpm}$ for $5 \mathrm{~min}$ and $0.45-\mu \mathrm{m}$ filtered. 500,000 THP-1 cells were transduced with $0.5 \mathrm{ml}$ lentiviral vectors, $8 \mu \mathrm{g} / \mathrm{ml}$ polybrene (Millipore), and $10 \mathrm{mM}$ Hepes (Invitrogen) in 12-well plates and medium replaced $24 \mathrm{~h}$ later. $2 \mathrm{~d}$ after transduction, transduced cells were selected with $0.5-8 \mu \mathrm{g} / \mathrm{ml}$ puromycin (Sigma-Aldrich). Cells were collected for analysis $4 \mathrm{~d}$ after transduction.

\section{Knockdown of gene expression by CRISPR}

Single guide RNAs targeting ATAD3A (oligonucleotides used given in Table 1) were designed using the CRISPOR tool (crispor.tefor.net) and cloned into lentiviral construct lentiCRISPRv2hygro, a gift from Brett Stringer (Flinders Health and Medical Research Institute, Adelaide, Australia; Stringer et al., 2019; plasmid \#98291; Addgene), following the protocol provided on the plasmid website. EVs 3 and 12 are two clones obtained after BSMBI digestion to remove buffer sequence, blunting and ligation using the Quick blunting kit (New England Biolabs), following the manufacturer's instructions. Lentiviral particles were produced, and THP-1 cells transduced as described for shRNA. $2 \mathrm{~d}$ after transduction, transduced cells were selected with $500 \mu \mathrm{g} / \mathrm{ml}$ hygromycin B (Invivogen), and selected cell pools were analyzed $7 \mathrm{~d}$ after transduction.

\section{Knockdown of gene expression by siRNA}

Expression of cGAS, MAVS, BAX, BAK1, ATAD3A, and TFAM in control or patient-derived primary fibroblasts was downregulated by using $20 \mathrm{nM}$ small interfering RNA (siRNA; references in Table 2). Negative control or gene-targeting siRNAs

Table 1. List of oligonucleotides for sgRNA cloning

\begin{tabular}{lll}
\hline Variation & Sense & Antisense \\
\hline sgATAD3A 7 & $5^{\prime}$-CACCGTGCCAGATTCAGG & $5^{\prime}$-AAACAGGACGCCCTGAAT \\
& GCGTCCT-3' & CTGGCAC-3' \\
\hline SgATAD3A & $5^{\prime}$-CACCGTGGCTCTTCGGCA & $5^{\prime}$-AAACTGTTAATGCCGAAG \\
26 & TTAACA-3' & AGCCAC-3' \\
\hline
\end{tabular}


were delivered to cells using the Lipofectamine RNAiMAX Transfection Reagent (Invitrogen) according to the manufacturer's instructions. Cells were analyzed $72 \mathrm{~h}$ after transfection.

\section{mtDNA depletion}

mtDNA depletion in THP-1 cells was induced by low-dose ethidium bromide $(100 \mathrm{ng} / \mathrm{ml})$ treatment in medium supplemented with $50 \mu \mathrm{g} / \mathrm{ml}$ uridine (Sigma-Aldrich) and $1 \mathrm{mM}$ sodium pyruvate (GIBCO) for 7-14 d before shRNA knockdown. In primary fibroblasts, mtDNA depletion was induced by $100 \mu \mathrm{M}$ ddC (Sigma-Aldrich) in medium supplemented with uridine and sodium pyruvate, as for THP-1 cells, for 7-14 $\mathrm{d}$ before analysis. To control for mtDNA depletion, total DNA from $0.5 \times 10^{6}$ cells was extracted using the DNeasy Blood and Tissue Kit (Qiagen) following the manufacturer's instructions. DNA concentrations were determined by photometry (Nanodrop) and $15 \mathrm{ng}, 7.5 \mathrm{ng}$, and $3.75 \mathrm{ng}$ DNA was used to perform quantitative PCR (qPCR) for the mitochondrial gene MT-COXII and the nuclear gene GAPDH (primers given in Table 3). qPCR was performed using Power SYBR Green (Invitrogen). Ratios of $2^{-\mathrm{CT}}$ for MT-COXII over GAPDH for the different DNA concentrations were averaged and fold of data in UT cells is shown in the figures.

\section{Generation of U2OS cells stably expressing STING}

The pMSCV-hygro plasmid carrying WT STING1 cDNA (\#102598; Addgene) was used in combination with packaging vector pCLAmpho (Novus) and envelope vector pCMV-VSV-G (plasmid \#8454; Addgene) to produce retroviral vectors as described for shRNA lentiviral vectors. 100,000 U2OS cells were transduced with $0.5 \mathrm{ml}$ retroviral vectors, $8 \mu \mathrm{g} / \mathrm{ml}$ polybrene (Millipore), and $10 \mathrm{mM}$ Hepes (Invitrogen) in 12-well plates and medium replaced $24 \mathrm{~h}$ later. $2 \mathrm{~d}$ after transduction, transduced cells were selected and maintained in culture with $200 \mu \mathrm{g} / \mathrm{ml}$ hygromycin

Table 2. List of siRNA references and sequences

\begin{tabular}{lll}
\hline Gene name & Assay ID/sequence & Manufacturer \\
\hline MB21D1 (cGAS) & s41746 & $\begin{array}{l}\text { Life } \\
\text { Technologies }\end{array}$ \\
\hline MAVS & 148763 & Life \\
& & Technologies \\
\hline BAX & 213259 & Life \\
\hline BAK1 & Technologies \\
\hline ATAD3A 1 (BJ-5ta) & s1881 & Life \\
\hline ATAD3A 2 (BJ-5ta) & T30447 & Technologies \\
\hline Negative control Med GC & 12935112 & Life \\
(siCTRL) & & Technologies \\
\hline $\begin{array}{l}\text { ATAD3A (Primary } \\
\text { fibroblasts) }\end{array}$ & SMARTpool 55210 & Life \\
\hline TFAM & & Technologies \\
\hline Scramble siRNA (siSCR) & 5'-UGCGCUAGGCCUCGG & Eurofins (siMAX) \\
\hline & UUGC-3' & Technologies \\
\hline
\end{tabular}

Lepelley et al.

Interferon signaling in a mitochondrial disease
Table 3. List of primers for DNA qPCR

\begin{tabular}{lll}
\hline Type & Primers & Sequence \\
\hline Sybr & MT-COXII_F & 5'-CGTCTGAACTATCCTGCCCG-3' $^{\prime}$ \\
\hline & MT-COXII_R & $5^{\prime}$-TGGTAAGGGAGGGATCGTTG-3' \\
\hline & GAPDH_F & $5^{\prime}$-ATGCTGCATTCGCCCTCTTA-3' \\
\hline & GAPDH_R & $5^{\prime}$-GCGCCCAATACGACCAAATC-3' \\
\hline
\end{tabular}

B (Invivogen). STING expression was verified by Western blotting.

\section{Overexpression of WT and mutant ATAD3A}

pCMV6-entry vector encoding Myc-DKK-tagged human WT ATAD3A cDNA (NM_018188.4, Q9NV17-1) was obtained from OriGene, and mutations were inserted by site-directed mutagenesis using the Q5 kit (E0554S; New England Biolabs) according to the manufacturer's instructions and using the primers listed in Table 4 . To obtain plasmids encoding the most common ATAD3A isoform (NM_001170535.2, Q9NV17-2), the sequence comprising the beginning of exon 3 was removed using the Q5 kit and primers below. pCMV6-Myc-DKK EV was generated by digestion with EcoRI and MluI restriction enzymes (Thermo Fischer Scientific), end blunting (Quick blunting kit; New England Biolabs), and ligation (T4 ligase; New England Biolabs), following the manufacturer's instructions. Constructs were verified by Sanger sequencing.

At 70\% confluency, U2OS-STING cells plated in 12-well plates were transiently transfected using TransIT-2020 (Mirus) with 500 ng ATAD3A WT, G355D, R528W, or WD cDNA in pCMV6Myc-DDK plasmid. $5 \mathrm{~h}$ after transfection, medium was changed, and $24 \mathrm{~h}$ after transfection, cells were treated with $10 \mu \mathrm{M} \mathrm{Q}-\mathrm{VD}$ OPh (SML0063; Sigma-Aldrich). Cells were collected 24 h later for RNA and protein analysis.

\section{SDS-PAGE and Western blot analysis}

For whole-cell lysate analysis, proteins were extracted using lysis buffer (radioimmunoprecipitation assay, 1\% protease inhibitor, and $1 \%$ phosphatase inhibitor). Bolt LDS Sample Buffer (4×; Novex Life Technologies) and Bolt Sample Reducing agent (10×; Novex Life Technologies) were added to protein lysates;

Table 4. List of primers for ATAD3A mutagenesis

\begin{tabular}{|c|c|c|}
\hline Variation & Forward primer & Reverse primer \\
\hline p.G355D & $\begin{array}{l}\text { 5'-GGGCCACCAGaCACC } \\
\text { GGGAAG-3' }\end{array}$ & $\begin{array}{l}\text { 5'-GTACATCAGGATGTTCCT } \\
\text { GTACAGG-3' }\end{array}$ \\
\hline p.R528W & $\begin{array}{l}5^{\prime} \text {-CATGTCGGGCtGGGA } \\
\text { GATCGC-3' }\end{array}$ & $\begin{array}{l}\text { 5'-CCCTCCGTCAGCCGA } \\
\text { GCG-3' }\end{array}$ \\
\hline WD (p.K358A) & $\begin{array}{l}\text { 5'-AGGCACCGGGgcGAC } \\
\text { GCTGTTTG-3' }\end{array}$ & $\begin{array}{l}\text { 5'-GGTGGCCCGTACATC } \\
\text { AGG-3' }\end{array}$ \\
\hline $\begin{array}{l}\text { Exon } 3 \text { part } \\
\text { deletion }\end{array}$ & $\begin{array}{l}\text { 5'-GAGTATGAGGCCGCC } \\
\text { GTG-3' }\end{array}$ & $\begin{array}{l}\text { 5'-TTTGAGCTTGGACTGTTG } \\
\text { CTC-3' }\end{array}$ \\
\hline
\end{tabular}

Lowercase letters indicate mutated bases. 
samples were resolved on $8 \%$ or $4-12 \%$ Bis-Tris Plus gels (Invitrogen) and then transferred to nitrocellulose membrane for $7 \mathrm{~min}$ at $20 \mathrm{~V}$ using the iBlot 2 Dry Blotting System (Invitrogen). Where protein phosphorylation status was investigated, membranes were blocked in LI-COR buffer and primary phosphoantibodies incubated for $48 \mathrm{~h}$ in the blocking solution. Otherwise, membranes were blocked with $5 \%$ nonfat milk in Tris-buffered saline (TBS) and primary antibodies incubated overnight at $4^{\circ} \mathrm{C}$ in $1.5 \%$ bovine serum albumin in TBS buffer supplemented with $0.1 \%$ Tween for most antibodies, $2.5 \%$ nonfat milk in TBS buffer supplemented with $0.1 \%$ Tween for anti-STING antibody, and $1.5 \%$ bovine serum albumin in TBS buffer supplemented with $0.4 \%$ Tween for rabbit anti-ATAD3 antibodies. A list of antibodies used in this study is supplied in Table 5. Membranes were washed and incubated with appropriate anti-mouse or anti-rabbit secondary antibodies for $45 \mathrm{~min}$ at room temperature (LI-COR System). Signal was detected using the Odyssey CLx System (LI-COR). Comparative signal analyses were performed using Fiji (ImageJ).

\section{RT-qPCR quantification of gene expression}

Total RNA was extracted using the RNAqueous-Micro Kit (Ambion), and reverse transcription was performed with the High-Capacity cDNA Reverse Transcription Kit (Applied Biosystems). Levels of cDNA were quantified by RT-qPCR using TaqMan Gene Expression Assays (Applied Biosystems). Differences in CDNA inputs were corrected by normalization to HPRT1 cDNA levels. Relative quantitation of target cDNA was determined by the formula $2^{-\Delta \mathrm{CT}}$, with $\Delta \mathrm{CT}$ denoting fold increase above control. When indicated, an ISG score was calculated as the median of the fold change expression of RSAD2, OAS1, Mx1, IFI27, ISG15, IRF7, and IFI44L, unless otherwise stated, and used as a readout. A list of the probes used in this study is supplied in Table 6.

\section{DNA immunofluorescence staining and confocal microscopy}

BJ-5ta cells were grown on a glass coverslip in a 6-well plate $24 \mathrm{~h}$ before treatment with indicated siRNAs. $3 \mathrm{~d}$ after siRNA treatment, cells were labeled with Mitotracker Red CMXRos (100 $\mathrm{nM}$ ) for $30 \mathrm{~min}$. Cells were washed and fixed with $4 \%$ paraformaldehyde in PBS and permeabilized with $0.25 \%$ Triton X-100, followed by blocking in 3\% bovine serum albumin/PBS for $1 \mathrm{~h}$. Cells were incubated with primary antibody (mouse monoclonal anti-DNA [AC-30-10; Progene] used at 1:200 concentration) overnight in a humidified chamber at $4^{\circ} \mathrm{C}$ followed by extensive washing before addition of secondary antibody (goat anti-mouse IgG, IgM [H+L], Alexa Fluor 488 at 1:300 concentration) for $1 \mathrm{~h}$ at room temperature. Slides were mounted in Vectashield antifade aqueous mounting medium (Vector Laboratories) with DAPI. Single-plane images were unbiasedly acquired with Nikon AlR+ confocal microscope using a $60.0 \times / 1.4$ oil objective. Analysis was performed on single focal planes acquired. Images were processed and analyzed using ImageJ Fiji. Since the mitochondrial networks were similar between conditions, the number per cell of DNA-stained foci not overlayed with the mitochondrial stain, and thus cytosolic, was counted. Three to eight fields of view were analyzed per condition per experiment in a blinded fashion.

\section{Automated imaging of mitochondrial morphology}

Human fibroblasts cells were seeded on CellCarrier 96-well Ultra microplate (PerkinElmer) and incubated for at least $24 \mathrm{~h}$ in growth media. Nuclei were labeled with NucBlue Live ReadyProbes Reagent (Thermo Fisher Scientific). Fluorescent labeling of mitochondria was achieved using tetramethylrhodamine ethyl ester perchlorate and MitoTracker DeepRed at $100 \mathrm{nM}$ for $30 \mathrm{~min}$ at $37^{\circ} \mathrm{C}, 5 \% \mathrm{CO}_{2}$. Cells were treated with $5 \mu \mathrm{M}$ protonophore carbonyl cyanide m-chlorophenyl hydrazone (CCCP, Sigma-Aldrich) or $10 \mu \mathrm{M}$ cytosolic protein synthesis inhibitor cycloheximide (CHX; Sigma-Aldrich) to induce mitochondrial fragmentation and hypertubulation, respectively. Spinning-disc confocal images were acquired using the Operetta CLS HighContent Analysis systems (PerkinElmer), with $63 \times$ water/1.15 numerical aperture. Tetramethylrhodamine ethyl ester perchlorate (530-560 nm), MitoTracker DeepRed (615-645 nm), and DAPI (355-385 nm) were excited the appropriate LEDs (Operetta CLS). Harmony Analysis Software (PerkinElmer) was used for automated confocal image analysis as described in detail in Cretin et al. (2021). Z-projected images first undergo brightfield correction. Nuclei and cellular segmentation were defined using the "Find Nuclei" building block with the HOECHST 33342 channel and the "Find Cytoplasm" building block with the Alexa Fluor 633 Mitotracker DeepRed (mitochondria) channel. Mitochondrial network was analyzed using SER Texture properties (ridge, bright, edge, dark, valley, and spot), and the PhenoLOGIC supervised machine learning algorithm was used to identify the best properties able to segregate the three populations: normal, fragmented, and hypertubulated network. 81-104 cells of each control (normal: WT + DMSO at t $=0 \mathrm{~h}$; fragmented: WT + CCCP at $\mathrm{t}=3 \mathrm{~h}$; and hypertubulated: $\mathrm{WT}+\mathrm{CHX}$ at $\mathrm{t}=6 \mathrm{~h}$ ) were selected to feed the algorithm for training. Automatic single-cell classification of nontraining samples (i.e., unknowns) was performed by the supervised machine learning module.

\section{Quantification and statistical analysis}

Statistical analysis was conducted using GraphPad Prism 8 software. Data were tested for normal distribution of variables and displayed as means \pm SEM unless otherwise noted. Measurements between two groups were performed with a Student's $t$ test if normally distributed, or Mann-Whitney test otherwise. Groups of three or more were analyzed by ANOVA or KruskalWallis test or paired ANOVA or Friedman test. Grouped analyses were interrogated by two-way ANOVA with post-hoc multiple comparison tests. Values of $n$ repeats and statistical parameters for each experiment are reported in the figures and figure legends. $\mathrm{P}<0.05$ was considered significant.

\section{Clinical reports \\ Patient 1}

This female was born at term following a normal pregnancy. She demonstrated excessive sleepiness in the first 2 wk of life with feeding difficulties. Developmental delay was obvious by age 5 mo. Neopterin was raised (256 nmol/liter [normal, 7-65 nmol/ liter]) in CSF at age $2 \mathrm{yr}$. She had extremely blond hair (unlike her parents or half-siblings), absent eyelashes and eyebrows, and deep-set eyes. She was hypotonic centrally and subsequently 
Table 5. List of antibodies

\begin{tabular}{|c|c|c|c|}
\hline Antibodies & Host species & Source & Catalog reference \\
\hline Cofilin (loading control) & Rabbit monoclonal & Cell Signaling & \#5175 \\
\hline$\beta$-Actin (loading control) & Rabbit monoclonal & Cell Signaling & \#3700 \\
\hline Vinculin (loading control) & Mouse monoclonal & Santa Cruz & sc-73614 \\
\hline ATAD3 & Mouse monoclonal & Santa Cruz & sc-376185 \\
\hline ATAD3 & Rabbit polyclonal & Gift of J. Baudier & N-Ter (Gilquin et al., 2010) \\
\hline IRF3 & Rabbit monoclonal & Cell Signaling & $\# 11904$ \\
\hline Phospho-(Ser396)IRF3 & Rabbit monoclonal & Cell Signaling & $\# 4947$ \\
\hline STING & Mouse monoclonal & R\&D Systems & MAB7169 \\
\hline Phospho-(Ser366)STING & Rabbit monoclonal & Cell Signaling & \#19781S \\
\hline TFAM & Mouse monoclonal & Santa Cruz & sc-376672 \\
\hline TOM20 & Mouse monoclonal & Santa Cruz & sc- 17764 \\
\hline VDAC1 & Mouse monoclonal & Santa Cruz & sc-390996 \\
\hline ISG15 & Rabbit polyclonal & Abcam & ab14374 \\
\hline MX1 & Rabbit polyclonal & Abcam & ab95926 \\
\hline BAX & Rabbit polyclonal & GeneTex & GTX109683 \\
\hline BAK1 & Rabbit polyclonal & GeneTex & GTX100063 \\
\hline FLAG (M2) & Mouse monoclonal & Sigma-Aldrich & $\mathrm{F} 3165$ \\
\hline IRDye $800 \mathrm{CW}$ (secondary antibody) & Goat anti-mouse & Li-COR & $926-32210$ \\
\hline IRDye $800 \mathrm{CW}$ (secondary antibody) & Goat anti-rabbit & Li-COR & $926-32211$ \\
\hline IRDye 640RD (secondary antibody) & Goat anti-mouse & $\mathrm{Li}-\mathrm{COR}$ & $926-68070$ \\
\hline IRDye 640RD (secondary antibody) & Goat anti-rabbit & $\mathrm{Li}-\mathrm{COR}$ & $926-68071$ \\
\hline
\end{tabular}

exhibited profound global developmental delay, visual impairment, and variable dystonia. She developed seizures at $5 \mathrm{yr}$ of age, which have been difficult to manage, requiring ventilation on two occasions for status epilepticus. At the age of $9 \mathrm{yr}$, she demonstrates profound delay, rigid dystonia, and no useful motor function. Communication is limited to eye movements and facial expression. Her head circumference is on the 50th centile, and cranial MRI showed some high signal in the anterior temporal lobes and at the parietal ventricular poles, with no obvious calcification.

\section{Patient 2}

This female was noted to have an absence of the eyelashes and eyebrows, myopia, and early-onset alacrimia in the first year of life. She required corneal grafts at age $5 \mathrm{yr}$ because of bilateral keratitis secondary to her alacrimia. She subsequently developed features of autoimmunity including antibody-positive Hashimoto's thyroiditis and vitiligo, also demonstrating growth hormone deficiency. Mild lower limb spasticity was noted in infancy, prompting cerebral MRI at age $6 \mathrm{yr}$ and a CT $1 \mathrm{yr}$ later, which were both normal. She attended university, with no intellectual deficits into adulthood, but demonstrates a spastic diplegic gait, which appears stable. Beginning at $21 \mathrm{yr}$ of age, she developed sclerodermatous involvement of the hands, face, and ventral surface of the forearms, which progressed rapidly over a few months. When reviewed aged $22 \mathrm{yr}$, she exhibited small joint swelling and diffuse skin thickening, particularly involving the fingers of both hands and extending proximal to the metacarpophalangeal joints (Rodnan skin score: 33/51), fulfilling American College of Rheumatology/European League Against Rheumatism criteria for a diagnosis of systemic sclerosis. There was an absence of digital ulcerations. Immunoglobulin levels, antinuclear $(1 / 5,220)$ and anti-dsDNA antibody titers were persistently elevated. Systemic sclerosis-related antibodies were not detected. Skin biopsy of affected tissue showed classical features of systemic sclerosis, with massive collagen fibrosis, loss of elastic fibers and adnexal epithelia, and deep peri-vascular lymphocytic infiltrates in the absence of immunoglobulin or complement deposition. In view of the rapid clinical progression, oral corticosteroids were initiated, resulting in a prompt remission of arthralgia and joint swelling. However, given no apparent effect on skin thickening and hardening after 11 mo of treatment, rapamycin was introduced and was associated with a partial regression of the cutaneous fibrosis and a decrease of the Rodnan skin score to 10/51 over the following $2 \mathrm{yr}$.

\section{Patient 3}

This female demonstrated early-onset severe motor delay with impaired cognition but good social contact. Extensive genetic investigations were negative, including array comparative genomic hybridization. Within the first year of life, she developed feeding difficulties, with relatively rapid feeding noted to easily promote vomiting, eventually necessitating the placement of a gastrostomy at 3 yr of age. Respiratory chain enzyme analysis in 
Table 6. List of Taqman assays for cDNA qPCR

\begin{tabular}{lll}
\hline Type & Primers & Assay ID \\
\hline TaqMan & HPRT1 & Hs03929096_g1 \\
\hline IFI27 & Hs01086370_m1 \\
\hline IFI44L & Hs00199115_m1 \\
\hline IFIT1 & Hs00356631_g1 \\
\hline ISG15 & Hs00192713_m1 \\
\hline RSAD2 & Hs01057264_m1 \\
\hline SIGLEC1 & Hs00988063_m1 \\
\hline OAS1 & Hs00973637_m1 \\
\hline Mx1 & Hs00895608_m1 \\
\hline IRF7 & Hs01014809_g1 \\
\hline IFNB1 & Hs01077958_s1 \\
\hline IFNA2 & Hs00265051-s1 \\
\hline IFNG & Hs00989291-m1 \\
\hline TFAM & Hs00273372_s1 \\
\hline ATAD3A & Hs01587333_m1 \\
\hline MB21D1 (cGAS) & Hs00403553_m1 \\
\hline MAVS & Hs00920075_m1 \\
\hline BAK1 & Hs00832876_g1 \\
\hline &
\end{tabular}

fibroblasts was normal (Bird et al., 2019). From age 2 yr, she developed a progressive hardening of the skin, a change in facial appearance due to loss of fat tissue, and areas of panniculitis involving the lower limbs. At $4 \mathrm{yr}$ of age, she exhibited perioral sclerosis, hard and tight sclerodermatous skin of the upper and lower limbs, and severe flexion contractures at the knees, ankles, elbows, wrists, and fingers (Rodnan skin score: 32/51). Skin biopsy revealed deep dermal sclerosis and loss of skin appendages, thick collagen bundles, and mild lymphocytic infiltration. MRI of the upper limbs confirmed cutaneous thickening. Treatment with rapamycin and low-dose corticosteroids was initiated at age $4 \mathrm{yr}$, leading to resolution of nightly unrest and cessation of pain medication, reduced induration of sclerotic limb lesions, improved mouth closure, and the reintroduction of supplementary oral feeding.

\section{Patient 4}

This male, now aged $2 \mathrm{yr}$, demonstrates severe developmental delay with central hypotonia, peripheral dystonia, a peripheral neuropathy, and hypertrophic cardiomyopathy. There was a deficit of respiratory chain complex I and V on muscle biopsy and a lactate peak seen on cerebral MRI, both before the age of $1 \mathrm{yr}$. A cranial CT at age $2 \mathrm{yr}$ did not demonstrate any evidence of intracranial calcification.

\section{Patient 5}

This 7-yr-old male (patient II-I, family 3 in Harel et al., 2016) exhibits global developmental delay, with truncal hypotonia, and peripheral spasticity. There is an absence of the eyebrows, optic nerve pallor, and macular hypoplasia. An echocardiogram revealed hypertrophic cardiomyopathy, initially detected at age 3 mo. Electrophysiology identified a peripheral neuropathy. Additionally, he has growth hormone deficiency and demonstrates extensive vitiligo. Plasma lactate was intermittently elevated, and a lactate peak was seen on magnetic resonance spectroscopy.

\section{Patient 6}

This 2-yr-old female of Iranian ancestry (described in Hanes et al., 2020) required the removal of bilateral cataracts at age $3 \mathrm{mo}$. At 5 mo old, she demonstrated axial hypotonia and hyporeflexia. Neurophysiology defined a sensorimotor polyneuropathy with axonal features, which was confirmed on nerve and muscle biopsy. By 8 mo of age, developmental regression became apparent, and she was no longer able to sit unsupported and had stopped rolling. Cranial MRI was unremarkable at this time. She was diagnosed with profound bilateral sensorineural hearing loss. At age $18 \mathrm{mo}$, she presented with bilateral ophthalmoplegia, partial left-sided ptosis, and visual impairment, as well as upper and lower limb dystonic posturing that did not respond to levodopa-carbidopa. At 21 mo of age, she developed refractory epilepsy, and at age $24 \mathrm{mo}$, a tracheostomy was placed due to intermittent central and obstructive apneas and an inability to wean off mechanical ventilation. Repeat MRI showed progressive cerebral and cerebellar atrophy between studies performed at 20 and 24 mo of age. Magnetic resonance spectroscopy and repeated serum lactate measurements were normal. At age $32 \mathrm{mo}$, she remains ventilated via tracheostomy, with little interaction with her surroundings and ongoing seizures and dystonia.

\section{Patient 7}

This patient, also reported previously (Cooper et al., 2017), was noted to demonstrate bilateral lower limb spasticity resulting in Achilles tendon lengthening at an early age. Now, aged $40 \mathrm{yr}$, she exhibits severe spastic paraparesis. She is of normal intellect and performed well academically, her only other problem being myopia (minus 10 diopters) with significant photophobia resulting in restricted eye opening. Her son, also reported by Cooper et al. (2017), but not tested here, was noted at age $4 \mathrm{mo}$ to exhibit lower limb spasticity. Spinal and cranial MRI at 12 mo of age was normal. At the age of $3 \mathrm{yr}$, he demonstrated dyskinetic cerebral palsy with severe upper and lower limb spasticity and was unable to speak, sit, or stand but could crawl by pulling himself forward with his arms and roll on the floor. Similar to his mother, there is an absence of the lateral eyelashes and photophobia.

\section{Online supplemental material}

Fig. S1 shows clinical, histological, and imaging features of systemic sclerosis seen in patients 2 and 3 and a comparison of IFN scores in ATAD3A patients and AGS8/9 patients. Fig. S2 reports IFN induction upon ATAD3A down-regulation by CRISPR targeting in THP-1 cells and in patient PBMCs. Fig. S3 reports the study of the signaling pathways involved in IFN induction in patient fibroblasts. Table S1 summarizes molecular, clinical and IFN data relating to patients 1-7. Table S2 summarizes features related to systemic sclerosis in patients 2 and 3 . 


\section{Acknowledgments}

We sincerely thank Jacques Baudier (Institut de Biologie du Developpement de Marseille, Marseille, France) for the generous use of antibodies. The graphical abstract was created with BioRender.

Y.J. Crow acknowledges that this project has received funding from the European Research Council under the European Union's Horizon 2020 research and innovation program (grant agreement 786142), a state subsidy managed by the National Research Agency (France) under the "Investments for the Future" program bearing the reference ANR-10-IAHU-01, and the National Institute for Health Research UK Rare Genetic Disease Research Consortium. The project was supported by MSDAVENIR (Devo-Decode Project). E. Van Nieuwenhove acknowledges the Research Foundation Flanders (Fonds voor Wetenschappelijk Onderzoek Vlaanderen, grant 1S22716N). B. Callewaert is a Senior Clinical Investigator of the Research Foundation Flanders. Ghent University Hospital, University Hospital Leuven, and Hôpital Universitaire Necker are members of the European Reference Network on Skin Disorders. This project has received funding from the European Union's Horizon 2020 research and innovation program under Marie Skłodowska-Curie grant agreement 892311 (A. Lepelley).

Author contributions: Conceptualization, A. Lepelley, B. Bader-Meunier, C. Wouters, and Y.J. Crow; methodology, A. Lepelley, B. Bader-Meunier, C. Wouters, T. Wai, and Y.J. Crow; investigation, A. Lepelley, E. Della Mina, E. Van Nieuwenhove, L. Waumans, S. Fraitag, G.I. Rice, A. Dhir, M.L. Frémond, M.P. Rodero, L. Seabra, E. Carter, C. Bodemer, D. Buhas, B. Callewaert, P. de Lonlay, L. De Somer, D.A. Dyment, F. Faes, L. Grove, S. Holden, M. Hully, H.J. McMillan, M.A. Kurian, K. Suetens, H. Tyynismaa, S. Chhun, T. Wai, C. Wouters, and B. Bader-Meunier; writing - original draft, Y.J. Crow; writing - review \& editing, A. Lepelley, B. Bader-Meunier, C. Wouters, and Y.J. Crow; funding acquisition, Y.J. Crow; Supervision, A. Lepelley and Y.J. Crow.

Disclosures: E. Van Nieuwenhove reported grants from FWO/ Research Foundation Flanders (SB grant 1S22718N) during the conduct of the study. No other disclosures were reported.

Submitted: 23 July 2020

Revised: 14 December 2020

Accepted: 15 July 2021

\section{References}

Ah Kioon, M.D., C. Tripodo, D. Fernandez, K.A. Kirou, R.F. Spiera, M.K. Crow, J.K. Gordon, and F.J. Barrat. 2018. Plasmacytoid dendritic cells promote systemic sclerosis with a key role for TLR8. Sci. Transl. Med. 10: eaam8458. https://doi.org/10.1126/scitranslmed.aam8458

Barrat, F.J., K.B. Elkon, and K.A. Fitzgerald. 2016. Importance of Nucleic Acid Recognition in Inflammation and Autoimmunity. Annu. Rev. Med. 67: 323-336. https://doi.org/10.1146/annurev-med-052814-023338

Baudier, J. 2018. ATAD3 proteins: brokers of a mitochondria-endoplasmic reticulum connection in mammalian cells. Biol. Rev. Camb. Philos. Soc. 93:827-844. https://doi.org/10.1111/brv.12373

Bhattacharyya, S., J. Wei, and J. Varga. 2011. Understanding fibrosis in systemic sclerosis: shifting paradigms, emerging opportunities. Nat. Rev. Rheumatol. 8:42-54. https://doi.org/10.1038/nrrheum.2011.149
Bird, M.J., I. Adant, P. Windmolders, I. Vander Elst, C. Felgueira, R. Altassan, S.C. Gruenert, B. Ghesquière, P. Witters, D. Cassiman, and P. Vermeersch. 2019. Oxygraphy Versus Enzymology for the Biochemical Diagnosis of Primary Mitochondrial Disease. Metabolites. 9:220. https:// doi.org/10.3390/metabo9100220

Brkic, Z., L. van Bon, M. Cossu, C.G. van Helden-Meeuwsen, M.C. Vonk, H Knaapen, W. van den Berg, V.A. Dalm, P.L. Van Daele, A. Severino, et al. 2016. The interferon type I signature is present in systemic sclerosis before overt fibrosis and might contribute to its pathogenesis through high BAFF gene expression and high collagen synthesis. Ann. Rheum. Dis. 75:1567-1573. https://doi.org/10.1136/annrheumdis-2015-207392

Chen, H., H. Chen, J. Zhang, Y. Wang, A. Simoneau, H. Yang, A.S. Levine, L. Zou, Z. Chen, and L. Lan. 2020. cGAS suppresses genomic instability as a decelerator of replication forks. Sci. Adv. 6:eabb8941. https://doi.org/ 10.1126/sciadv.abb8941

Cooper, H.M., Y. Yang, E. Ylikallio, R. Khairullin, R. Woldegebriel, K.L. Lin, L. Euro, E. Palin, A. Wolf, R. Trokovic, et al. 2017. ATPase-deficient mitochondrial inner membrane protein ATAD3A disturbs mitochondrial dynamics in dominant hereditary spastic paraplegia. Hum. Mol. Genet. 26:1432-1443. https://doi.org/10.1093/hmg/ddx042

Cretin, E., P. Lopes, E. Vimont, T. Tatsuta, T. Langer, A. Gazi, M. Sachse, P. Yu-Wai-Man, P. Reynier, and T. Wai. 2021. High-throughput screening identifies suppressors of mitochondrial fragmentation in OPA1 fibroblasts. EMBO Mol. Med. 13:e13579. https://doi.org/10.15252/emmm 202013579

Crow, M.K. 2019. Mitochondrial DNA promotes autoimmunity. Science. 366: 1445-1446. https://doi.org/10.1126/science.aaz9308

Crow, Y.J., and N. Manel. 2015. Aicardi-Goutières syndrome and the type I interferonopathies. Nat. Rev. Immunol. 15:429-440. https://doi.org/10 $.1038 / \mathrm{nri} 3850$

Crow, Y.J., M.S. Zaki, M.S. Abdel-Hamid, G. Abdel-Salam, O. BoespflugTanguy, N.J. Cordeiro, J.G. Gleeson, N.R. Gowrinathan, V. Laugel, F. Renaldo, et al. 2014. Mutations in ADAR1, IFIH1, and RNASEH2B presenting as spastic paraplegia. Neuropediatrics. 45:386-393. https://doi .org/10.1055/s-0034-1389161

Crow, Y.J., D.S. Chase, J. Lowenstein Schmidt, M. Szynkiewicz, G.M. Forte, H.L. Gornall, A. Oojageer, B. Anderson, A. Pizzino, G. Helman, et al. 2015. Characterization of human disease phenotypes associated with mutations in TREX1, RNASEH2A, RNASEH2B, RNASEH2C, SAMHD1, ADAR, and IFIH1. Am. J. Med. Genet. A. 167A:296-312. https://doi.org/10 .1002/ajmg.a.36887

Desai, R., A.E. Frazier, R. Durigon, H. Patel, A.W. Jones, I. Dalla Rosa, N.J. Lake, A.G. Compton, H.S. Mountford, E.J. Tucker, et al. 2017. ATAD3 gene cluster deletions cause cerebellar dysfunction associated with altered mitochondrial DNA and cholesterol metabolism. Brain. 140: 1595-1610. https://doi.org/10.1093/brain/awx094

Dhir, A., S. Dhir, L.S. Borowski, L. Jimenez, M. Teitell, A. Rötig, Y.J. Crow, G.I. Rice, D. Duffy, C. Tamby, et al. 2018. Mitochondrial double-stranded RNA triggers antiviral signalling in humans. Nature. 560:238-242. https://doi.org/10.1038/s41586-018-0363-0

Dorison, N., P. Gaignard, A. Bayot, A. Gelot, P.H. Becker, S. Fourati, E. Lebigot, P. Charles, T. Wai, P. Therond, and A. Slama. 2020. Mitochondrial dysfunction caused by novel ATAD3A mutations. Mol. Genet. Metab. 131: 107-113. https://doi.org/10.1016/j.ymgme.2020.09.002

Evans, K.J., E.R. Gomes, S.M. Reisenweber, G.G. Gundersen, and B.P. Lauring 2005. Linking axonal degeneration to microtubule remodeling by Spastin-mediated microtubule severing. J. Cell Biol. 168:599-606. https://doi.org/10.1083/jcb.200409058

Frazier, A.E., A.G. Compton, Y. Kishita, D.H. Hock, A.E. Welch, S.S.C Amarasekera, R. Rius, L.E. Formosa, A. Imai-Okazaki, D. Francis, et al. 2021. Fatal perinatal mitochondrial cardiac failure caused by recurrent de novo duplications in the ATAD3 locus. $\operatorname{Med}(N$ Y). 2:49-73.

Gilquin, B., E. Taillebourg, N. Cherradi, A. Hubstenberger, O. Gay, N. Merle, N. Assard, M.O. Fauvarque, S. Tomohiro, O. Kuge, and J. Baudier. 2010. The AAA+ ATPase ATAD3A controls mitochondrial dynamics at the interface of the inner and outer membranes. Mol. Cell. Biol. 30: 1984-1996. https://doi.org/10.1128/MCB.00007-10

Gunning, A.C., K. Strucinska, M. Muñoz Oreja, A. Parrish, R. Caswell, K.L. Stals, R. Durigon, K. Durlacher-Betzer, M.H. Cunningham, C.M. Grochowski, et al. 2020. Recurrent De Novo NAHR Reciprocal Duplications in the ATAD3 Gene Cluster Cause a Neurogenetic Trait with Perturbed Cholesterol and Mitochondrial Metabolism. Am. J. Hum. Genet. 106: 272-279. https://doi.org/10.1016/j.ajhg.2020.01.007

Hanes, I., H.J. McMillan, Y. Ito, K.D. Kernohan, J. Lazier, M.A. Lines, and D.A. Dyment. 2020. A splice variant in ATAD3A expands the clinical and 
genetic spectrum of Harel-Yoon syndrome. Neurol. Genet. 6:e452. https://doi.org/10.1212/NXG.0000000000000452

Harel, T., W.H. Yoon, C. Garone, S. Gu, Z. Coban-Akdemir, M.K. Eldomery, J.E. Posey, S.N. Jhangiani, J.A. Rosenfeld, M.T. Cho, et al. University of Washington Center for Mendelian Genomics. 2016. Recurrent De Novo and Biallelic Variation of ATAD3A, Encoding a Mitochondrial Membrane Protein, Results in Distinct Neurological Syndromes. Am. J. Hum. Genet. 99:831-845. https://doi.org/10.1016/j.ajhg.2016.08.007

He, J., H.M. Cooper, A. Reyes, M. Di Re, H. Sembongi, T.R. Litwin, J. Gao, K.C. Neuman, I.M. Fearnley, A. Spinazzola, et al. 2012. Mitochondrial nucleoid interacting proteins support mitochondrial protein synthesis. Nucleic Acids Res. 40:6109-6121. https://doi.org/10.1093/nar/gks266

Kim, J., R. Gupta, L.P. Blanco, S. Yang, A. Shteinfer-Kuzmine, K. Wang, J. Zhu, H.E. Yoon, X. Wang, M. Kerkhofs, et al. 2019. VDAC oligomers form mitochondrial pores to release mtDNA fragments and promote lupuslike disease. Science. 366:1531-1536. https://doi.org/10.1126/science .aav4011

Lepelley, A., M.J. Martin-Niclós, M. Le Bihan, J.A. Marsh, C. Uggenti, G.I. Rice, V. Bondet, D. Duffy, J. Hertzog, J. Rehwinkel, et al. 2020. Mutations in COPA lead to abnormal trafficking of STING to the Golgi and interferon signaling. J. Exp. Med. 217:e20200600. https://doi.org/10.1084/jem .20200600

Li, Q., T. Zhang, J. Wang, Z. Zhang, Y. Zhai, G.Y. Yang, and X. Sun. 2014. Rapamycin attenuates mitochondrial dysfunction via activation of mitophagy in experimental ischemic stroke. Biochem. Biophys. Res. Commun. 444:182-188. https://doi.org/10.1016/j.bbrc.2014.01.032

Livingston, J.H., J.P. Lin, R.C. Dale, D. Gill, P. Brogan, A. Munnich, M.A Kurian, V. Gonzalez-Martinez, C.G. De Goede, A. Falconer, et al. 2014. A type I interferon signature identifies bilateral striatal necrosis due to mutations in ADAR1. J. Med. Genet. 51:76-82. https://doi.org/10.1136/ jmedgenet-2013-102038

López-Isac, E., M. Acosta-Herrera, M. Kerick, S. Assassi, A.T. Satpathy, J. Granja, M.R. Mumbach, L. Beretta, C.P. Simeón, P. Carreira, et al. Australian Scleroderma Interest Group (ASIG). 2019. GWAS for systemic sclerosis identifies multiple risk loci and highlights fibrotic and vasculopathy pathways. Nat. Commun. 10:4955. https://doi.org/10.1038/ s41467-019-12760-y

McArthur, K., L.W. Whitehead, J.M. Heddleston, L. Li, B.S. Padman, V. Oorschot, N.D. Geoghegan, S. Chappaz, S. Davidson, H. San Chin, et al. 2018. BAK/BAX macropores facilitate mitochondrial herniation and mtDNA efflux during apoptosis. Science. 359:eaao6047. https://doi.org/ 10.1126/science.aao6047

Muskardin, T.L.W., and T.B. Niewold. 2018. Type I interferon in rheumatic diseases. Nat. Rev. Rheumatol. 14:214-228. https://doi.org/10.1038/ nrrheum.2018.31

Peralta, S., A. González-Quintana, M. Ybarra, A. Delmiro, R. Pérez-Pérez, J. Docampo, J. Arenas, A. Blázquez, C. Ugalde, and M.A. Martín. 2019. Novel ATAD3A recessive mutation associated to fatal cerebellar hypoplasia with multiorgan involvement and mitochondrial structural abnormalities. Mol. Genet. Metab. 128:452-462. https://doi.org/10.1016/j .ymgme.2019.10.012

Rice, G.I., G.M. Forte, M. Szynkiewicz, D.S. Chase, A. Aeby, M.S. Abdel-Hamid, S. Ackroyd, R. Allcock, K.M. Bailey, U. Balottin, et al. 2013. Assessment of interferon-related biomarkers in Aicardi-Goutières syndrome associated with mutations in TREX1, RNASEH2A, RNASEH2B, RNASEH2C, SAMHD1, and ADAR: a case-control study. Lancet Neurol. 12:1159-1169. https://doi.org/10.1016/S1474-4422(13)70258-8

Rice, G.I., S. Park, F. Gavazzi, L.A. Adang, L.A. Ayuk, L. Van Eyck, L. Seabra, C. Barrea, R. Battini, A. Belot, et al. 2020. Genetic and phenotypic spectrum associated with IFIH1 gain-of-function. Hum. Mutat. 41:837-849. https://doi.org/10.1002/humu.23975

Riley, J.S., G. Quarato, C. Cloix, J. Lopez, J. O’Prey, M. Pearson, J. Chapman, H. Sesaki, L.M. Carlin, J.F. Passos, et al. 2018. Mitochondrial inner membrane permeabilisation enables mtDNA release during apoptosis. EMBO J. 37:e99238. https://doi.org/10.15252/embj.201899238

Rongvaux, A., R. Jackson, C.C. Harman, T. Li, A.P. West, M.R. de Zoete, Y. Wu, B. Yordy, S.A. Lakhani, C.Y. Kuan, et al. 2014. Apoptotic caspases prevent the induction of type I interferons by mitochondrial DNA. Cell. 159:1563-1577. https://doi.org/10.1016/j.cell.2014.11.037

Skaug, B., and S. Assassi. 2020. Type I interferon dysregulation in Systemic Sclerosis. Cytokine. 132:154635. https://doi.org/10.1016/j.cyto.2018.12 .018

Stringer, B.W., B.W. Day, R.C.J. D’Souza, P.R. Jamieson, K.S. Ensbey, Z.C. Bruce, Y.C. Lim, K. Goasdoué, C. Offenhäuser, S. Akgül, et al. 2019. A reference collection of patient-derived cell line and xenograft models of proneural, classical and mesenchymal glioblastoma. Sci. Rep. 9:4902. https://doi.org/10.1038/s41598-019-41277-z

Uggenti, C., A. Lepelley, and Y.J. Crow. 2019. Self-Awareness: Nucleic AcidDriven Inflammation and the Type I Interferonopathies. Annu. Rev. Immunol. 37:247-267. https://doi.org/10.1146/annurev-immunol-042718 $-041257$

Uggenti, C., A. Lepelley, M. Depp, A.P. Badrock, M.P. Rodero, M.T. El-Daher, G.I. Rice, S. Dhir, A.P. Wheeler, A. Dhir, et al. 2020. cGAS-mediated induction of type I interferon due to inborn errors of histone pre-mRNA processing. Nat. Genet. 52:1364-1372. https://doi.org/10.1038/s41588 $-020-00737-3$

Hertzog, J., W. Zhou, R.E. Rigby, G. Fowler, C. Cursi, L. Chauveau, T. Davenne, P.J. Kranzusch, and J. Rehwinkel. 2021. Varicella-Zoster Virus ORF9 Is an Antagonist of the DNA Sensor cGAS. bioRxiv. https://doi.org/10.1101/ 2020.02.11.943415 (Preprint posted April 18, 2021)

van den Hoogen, F., D. Khanna, J. Fransen, S.R. Johnson, M. Baron, A. Tyndall, M. Matucci-Cerinic, R.P. Naden, T.A. Medsger Jr., P.E. Carreira, et al. 2013. 2013 classification criteria for systemic sclerosis: an American College of Rheumatology/European League against Rheumatism collaborative initiative. Arthritis Rheum. 65:2737-2747. https://doi.org/10 .1002/art.38098

Van Eyck, L., L. De Somer, D. Pombal, S. Bornschein, G. Frans, S. HumbletBaron, L. Moens, F. de Zegher, X. Bossuyt, C. Wouters, and A. Liston. 2015. Brief Report: IFIH1 Mutation Causes Systemic Lupus Erythematosus With Selective IgA Deficiency. Arthritis Rheumatol. 67:1592-1597. https://doi.org/10.1002/art.39110

Villanueva Paz, M., D. Cotán, J. Garrido-Maraver, M.D. Cordero, M. OropesaÁvila, M. de La Mata, A. Delgado Pavón, I. de Lavera, E. Alcocer-Gómez, and J.A. Sánchez-Alcázar. 2016. Targeting autophagy and mitophagy for mitochondrial diseases treatment. Expert Opin. Ther. Targets. 20: 487-500. https://doi.org/10.1517/14728222.2016.1101068

Wang, T., Y. Jiao, and X. Zhang. 2021. Immunometabolic Pathways and Its Therapeutic Implication in Autoimmune Diseases. Clin. Rev. Allergy Immunol. 60:55-67. https://doi.org/10.1007/s12016-020-08821-6

West, A.P., and G.S. Shadel. 2017. Mitochondrial DNA in innate immune responses and inflammatory pathology. Nat. Rev. Immunol. 17:363-375. https://doi.org/10.1038/nri.2017.21

West, A.P., W. Khoury-Hanold, M. Staron, M.C. Tal, C.M. Pineda, S.M. Lang, M. Bestwick, B.A. Duguay, N. Raimundo, D.A. MacDuff, et al. 2015 Mitochondrial DNA stress primes the antiviral innate immune response. Nature. 520:553-557. https://doi.org/10.1038/nature14156

Wu, M., B. Skaug, X. Bi, T. Mills, G. Salazar, X. Zhou, J. Reveille, S.K. Agarwal, M.R. Blackburn, M.D. Mayes, and S. Assassi. 2019. Interferon regulatory factor 7 (IRF7) represents a link between inflammation and fibrosis in the pathogenesis of systemic sclerosis. Ann. Rheum. Dis. 78:1583-1591. https://doi.org/10.1136/annrheumdis-2019-215208

Yu, C.H., S. Davidson, C.R. Harapas, J.B. Hilton, M.J. Mlodzianoski, P. Laohamonthonkul, C. Louis, R.R.J. Low, J. Moecking, D. De Nardo, et al. 2020. TDP-43 Triggers Mitochondrial DNA Release via mPTP to Activate cGAS/STING in ALS. Cell. 183:636-649.e18. https://doi.org/10.1016/ j.cell.2020.09.020

Zhao, Y., X. Sun, D. Hu, D.A. Prosdocimo, C. Hoppel, M.K. Jain, R. Ramachandran, and X. Qi. 2019. ATAD3A oligomerization causes neurodegeneration by coupling mitochondrial fragmentation and bioenergetics defects. Nat. Commun. 10:1371. https://doi.org/10 1038/s41467-019-09291-x

Zhou, R., A.S. Yazdi, P. Menu, and J. Tschopp. 2011. A role for mitochondria in NLRP3 inflammasome activation. Nature. 469:221-225. https://doi.org/ $10.1038 /$ nature09663 


\section{Supplemental material}

A Features of systemic sclerosis in patients with mutations in ATAD3A
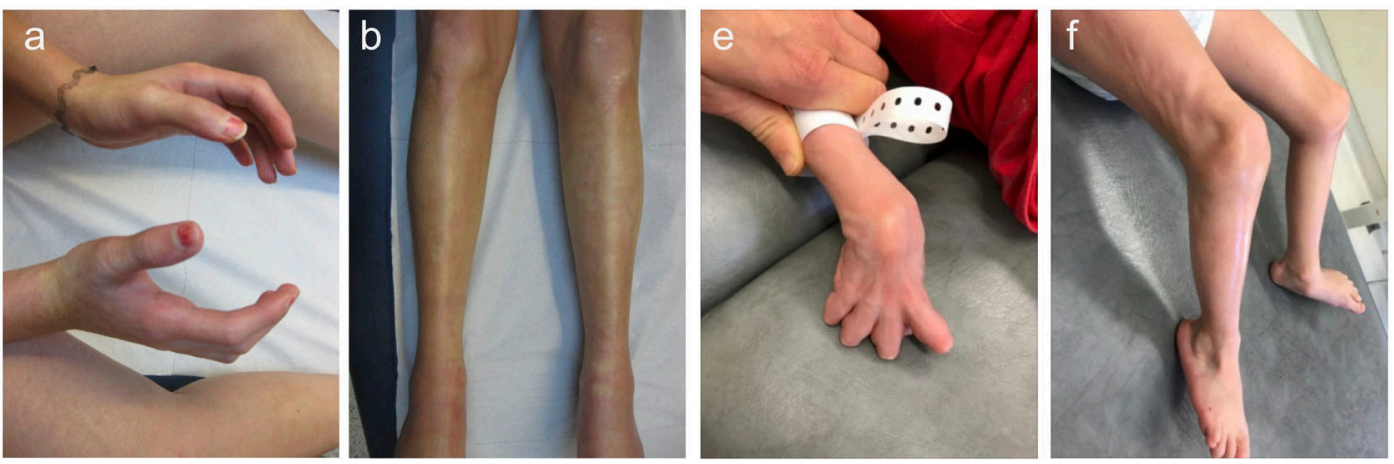

\section{B Histological features of skin biopsies}
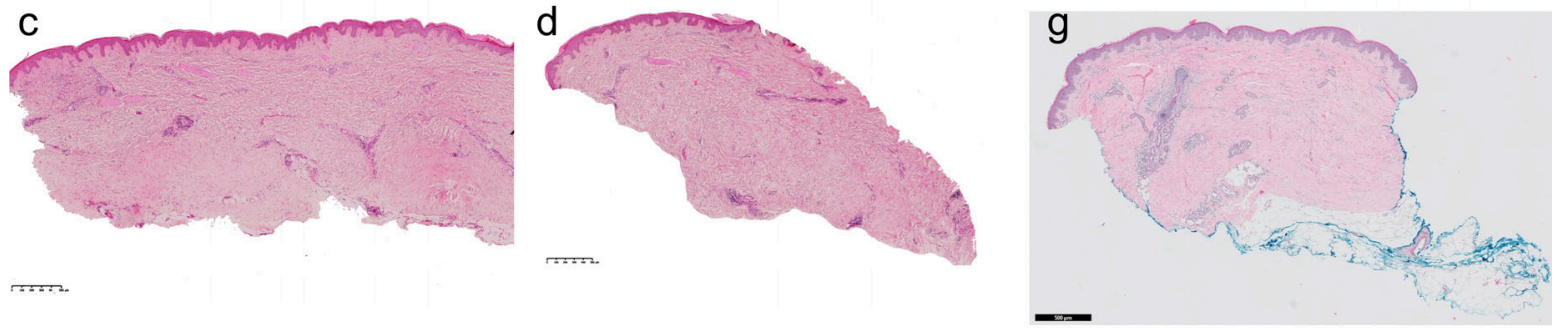

\section{MRI characteristics}

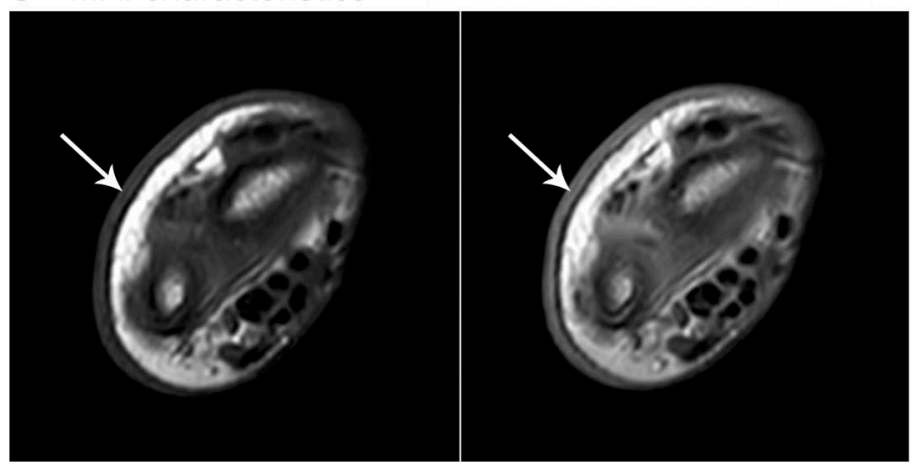

D Interferon score

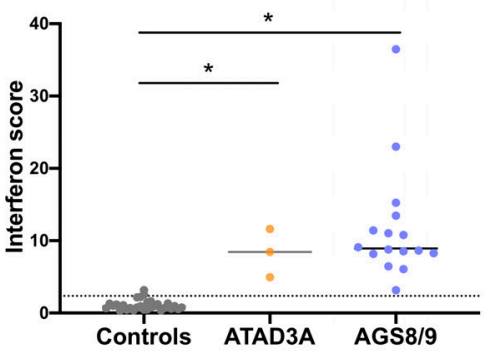

Figure S1. Clinical, histological, and imaging features of systemic sclerosis seen in patients 2 and 3 and IFN score in ATAD3A and AGS8/9 patients. (A) Clinical features of systemic sclerosis seen in patients 2 and 3. Subpanels a and b highlight sclerodermatous involvement of the hands and legs of patient 2 at 22 yr of age. Similar features were observed in patient 3 at age 4 yr (subpanels e and f). (B) Features of systemic sclerosis seen on skin biopsy. Subpanels c, $\mathrm{d}$, and $\mathrm{g}$ demonstrate the appearances on skin biopsy observed in patients 2 and 3 , respectively. In subpanel c, the overlying epidermis is normal, while the dermis is increased in thickness and composed of broad sclerotic collagen bundles extending into the subcutis and replacing fat. The deep dermis shows homogenized collagen and is devoid of elastic tissue. In addition, eccrine glands are very atrophic. The arrectores pilorum of the hair follicles are only recognizable in the upper part of the dermis, because the epithelium is almost completely absent. Subpanel $d$ is from another part of the biopsy, showing the same changes with, in addition, perivascular lymphocytic infiltrate in the deep dermis. In subpanel g, similar changes are seen to those observed in patient 2, but with skin adnexae (hair follicles and sweat glands) still present, consistent with an earlier stage in the same disease spectrum. Scale bars, $500 \mu \mathrm{m}$. (C) MRI of right forearm of patient 3. Left: Turbo spin-echo T1 sequence before contrast shows diffuse cutaneous thickening in the right forearm, most pronounced on the dorsal-ulnar aspect. Right: Turbo spin-echo T1 sequence after contrast administration (Dotarem $0.5 \mathrm{mmol} / \mathrm{ml}$ ). Arrows indicate signal in the thickened cutis before and after enhancement. (D) Dot plot showing the IFN score calculated from the median fold change of six ISGs measured in blood of ATAD3A patients 1, 2, and 3 and AGS8/9 patients. Average of one to eight serial samples for each patient is represented. ${ }^{*}$ indicates statistical significance $(P<0.05)$ in a $t$ test. Data for AGS8/9 patients are from Uggenti et al. (2020). 

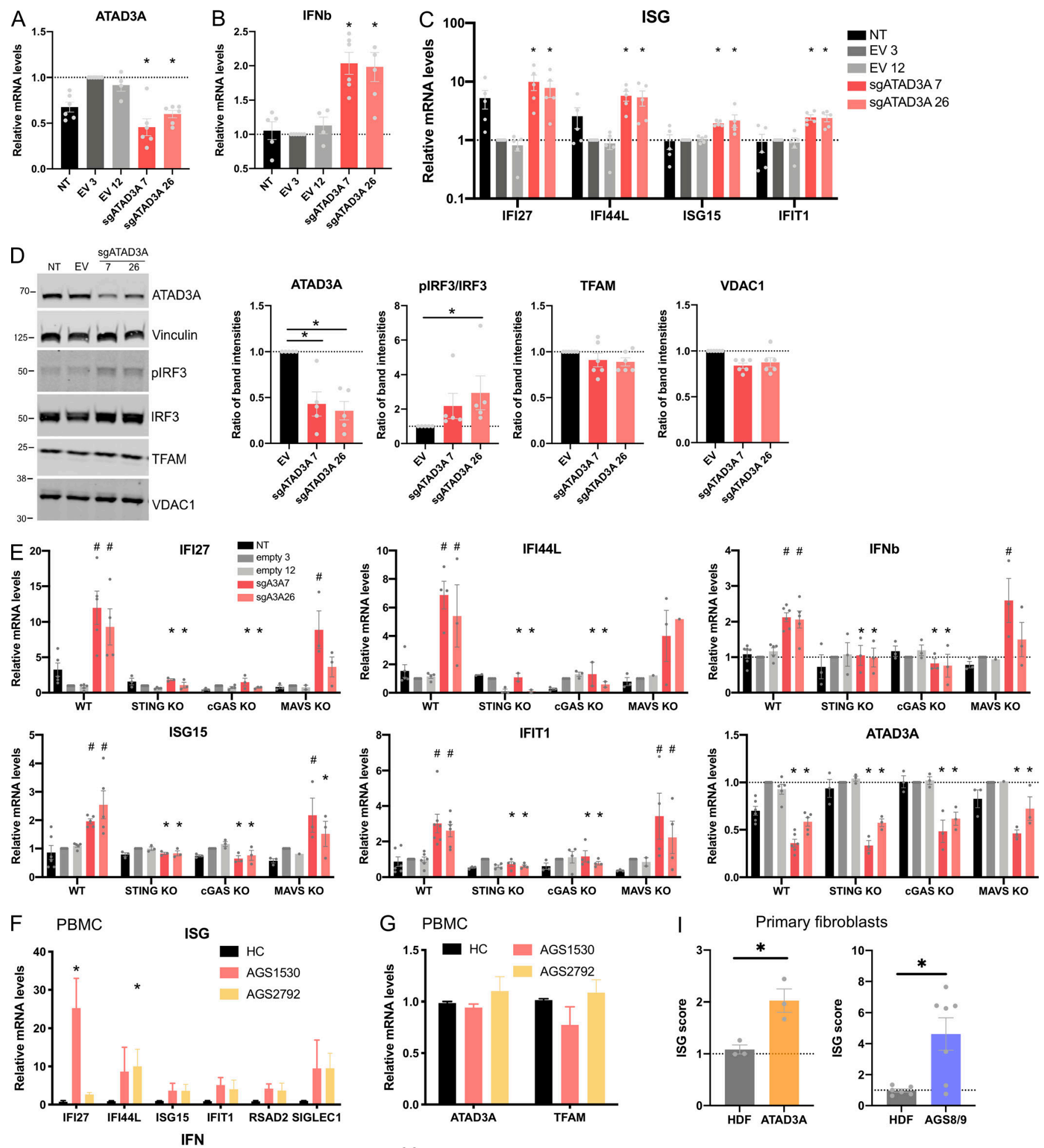

G PBMC

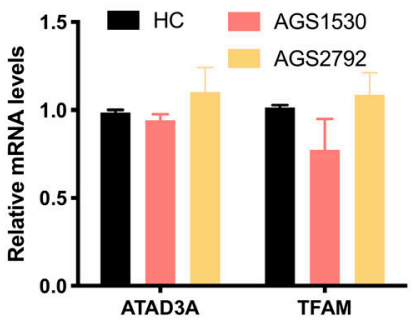

$\mathrm{H}$

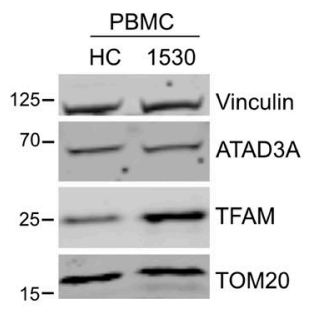

Primary fibroblasts
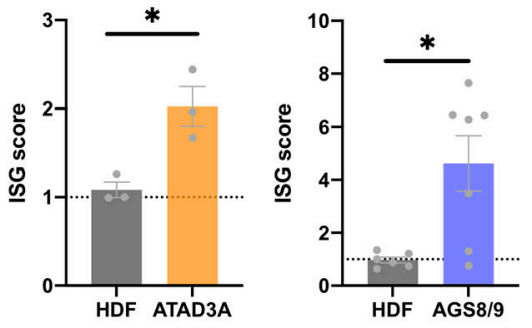

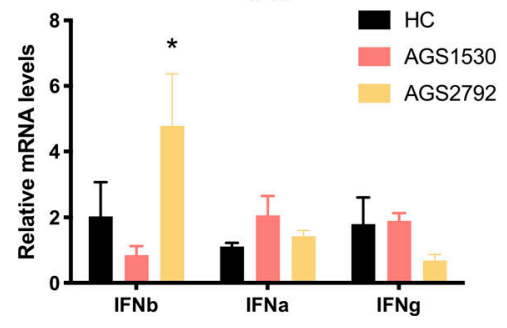


Figure S2. ATAD3A down-regulation by CRISPR editing in THP-1 cells and IFN signaling in ATAD3A and AGS8/9 patient primary cells. (A) qPCR of ATAD3A following knockdown of ATAD3A by CRISPR editing in THP-1 cell pools using two different guide RNAs (sgATAD3A 7, sgATAD3A 26) compared with NT cells and cells transduced with one of two EVs (EV 3 and 12). (B) Up-regulation of IFN- $\beta$ (IFNb) mRNA expression. (C) Up-regulation of ISG (IFI27, IFI44L, ISG15, and IFIT1) mRNA expression. Mean values of five independent experiments are shown and are expressed as the ratio of the mRNA levels, normalized to housekeeping gene HPRT mRNA, in indicated conditions to that in control EV 3. ${ }^{*}$ indicates significance $(P<0.05)$ using a Kruskal-Wallis test with Dunn's post hoc compared with EV 3 for each ISG. (D) Increased phosphorylated IRF3 (pIRF3; blot on the left, quantification on the right). The specificity of ATAD3A knockdown is indicated by an absence of a change in the expression of either of the mitochondrial proteins TFAM (matrix) or VDAC1 (outer membrane). Quantification of band intensities is expressed as phosphorylated IRF3/total IRF3 ratio and protein levels normalized to vinculin, averaged across four to five independent experiments; EV is EV 3. (E) IFN- $\beta$ (IFNb) and representative ISG (IFI27, IFI44L, ISG15, and IFITI) mRNA expression upon knockdown of ATAD3A with CRISPR editing as in A in WT THP-1 cells and in cells null (KO) for CGAS, STING, or MAVS. Mean values of three to five independent experiments are shown and are expressed as the ratio of the mRNA levels, normalized to housekeeping gene HPRT mRNA, in indicated conditions to that with EV 3. ${ }^{*}$ indicates statistical significance $(P<0.05)$ using two-way ANOVA with Dunnett's multiple comparison test between cell lines for each CRISPR condition. \# indicates statistical significance $(P<0.05)$ using two-way ANOVA with Dunnett's multiple comparison test between CRISPR conditions for each cell line. (F and $G)$ ISGs, IFN- $\beta$ (IFNb), IFN-a $2 a(I F N a)$, and IFN-y (IFNg; F) and ATAD3A and TFAM (G) mRNA expression in PBMCs isolated from healthy control (HC) donors or ATAD3A patients 2 and 4 (AGS1530 and AGS2792, respectively). Mean values and data points of two to three different samples are shown and are expressed as the ratio of the mRNA levels, normalized to housekeeping gene HPRT mRNA, in indicated conditions to that in one healthy control. * indicates statistical significance $(P<0.05)$ in two-way ANOVA with Dunnett's multiple comparison test to healthy controls for each probe. (H) Representative protein expression analysis of ATAD3A and TFAM expression in PBMCs by Western blot. TOM20 is an outer mitochondrial membrane protein, and vinculin a loading control. (I) ISG mRNA expression, represented as an ISG score (i.e., the median fold change of mRNA levels of six ISGs [RSAD2, OAS1, Mx1, IFI27, ISG15, and IFI44L] for ATAD3A or five ISGs [IFI27, IFI44L, IFIT1, ISG15, and RSAD2] for AGS8/9 in control and ATAD3A or AGS8/9 patient-derived primary fibroblasts. Summary mean value of 5-10 experiments for each cell line is shown. ${ }^{*}$ indicates statistical significance $(P<0.05)$ in a $t$ test. Data for AGS8/9 patients are from Uggenti et al. (2020). 


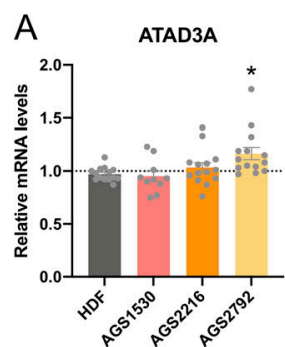

B HDF $\frac{\text { Patient }}{279222161530} \underline{\text { HDF }}$

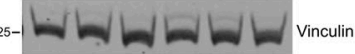

${ }^{70-} \sim \sim \sim \sim \sim-1 \mathrm{AAD} 3 \mathrm{~A}$

$32 \ldots \ldots \ldots$ VDAC1

C mtDNA copy number
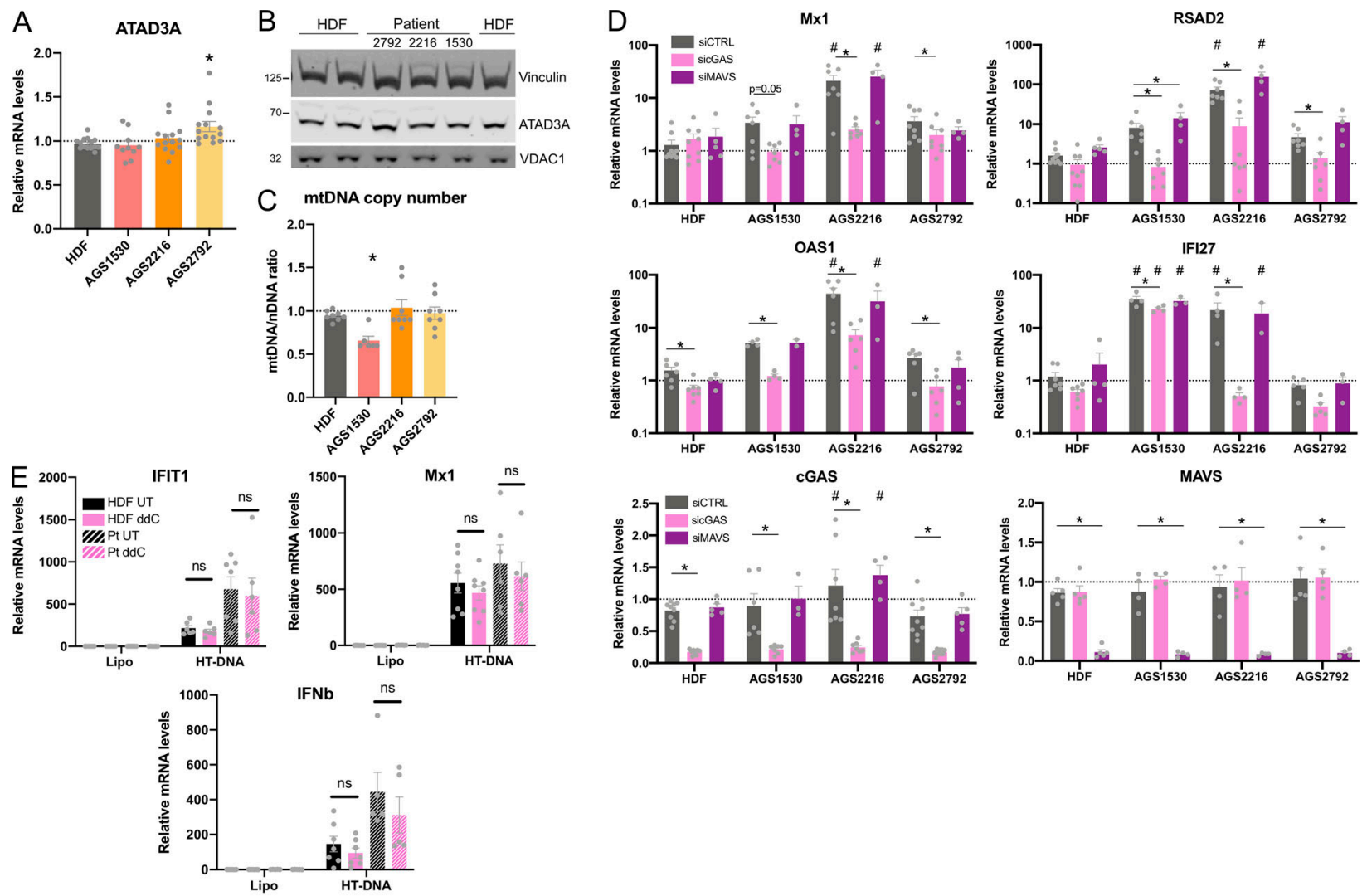

cGAS
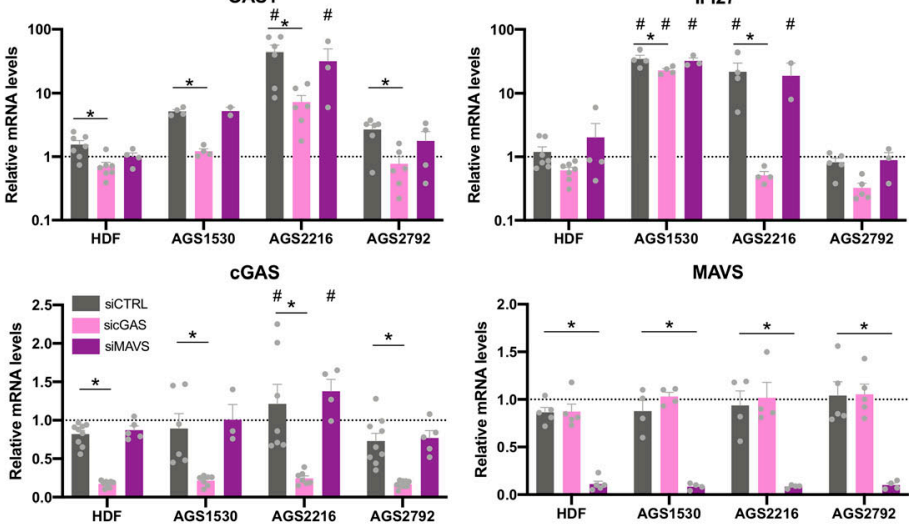

MAVS
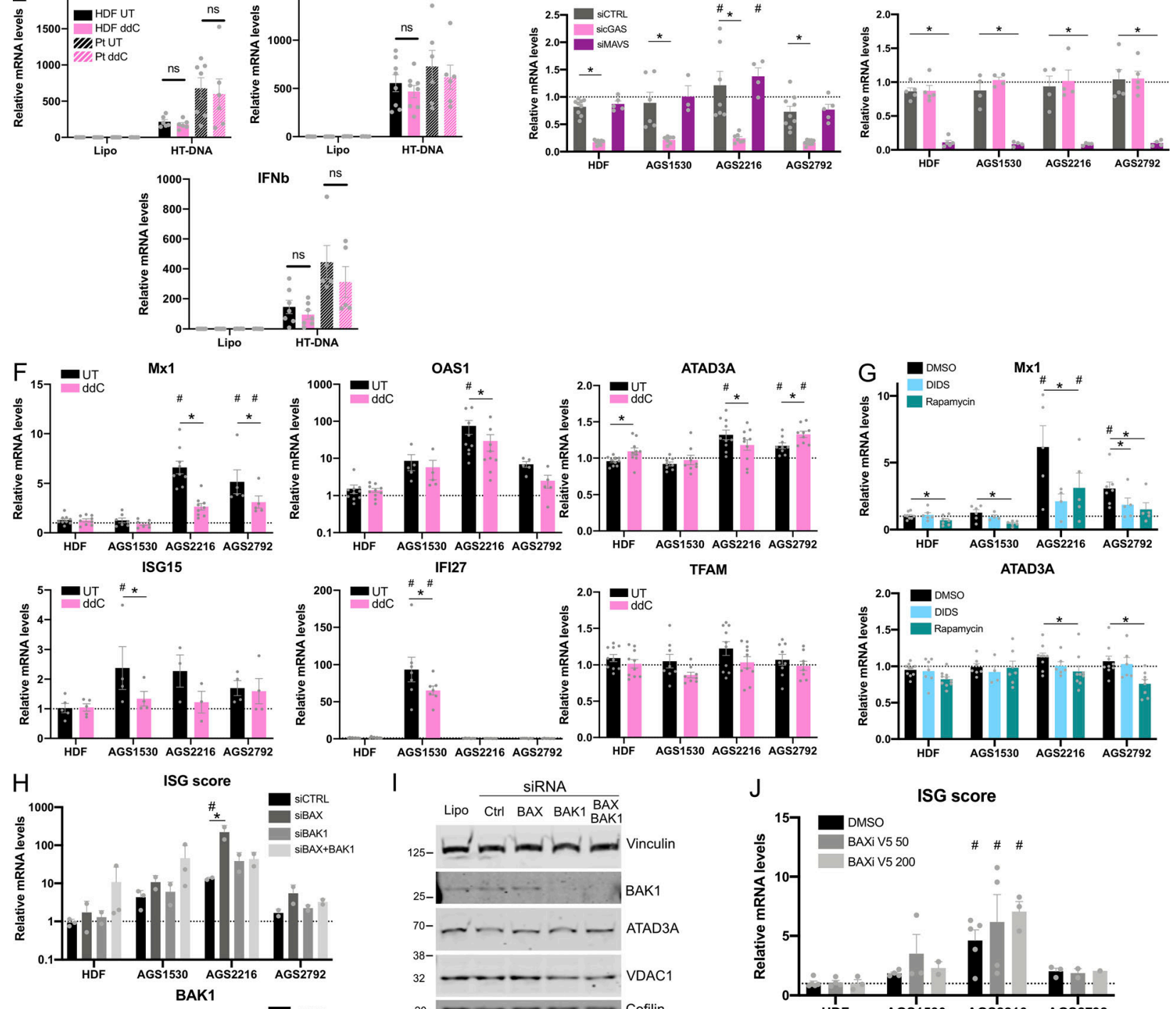

I

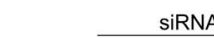

Lipo Ctrl BAX BAK1 $\underset{B A K 1}{B A X}$

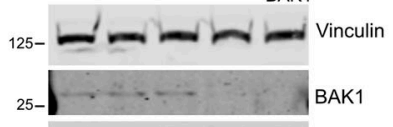

$70-\ldots \ldots$ ATAD3A
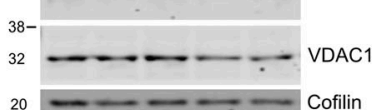

SiRNA

Lipo Ctrl BAX BAK1 ${ }_{\text {BAK1 }}^{\text {BAX }}$

25- $-\cdots$ BAK1 - BAX

$20-\square-\cdots$ Cofilin 
Figure S3. Characteristics of IFN signaling induction in ATAD3A patient-derived fibroblasts. (A) ATAD3A mRNA expression in primary fibroblasts from controls (HDF; average of three) or from ATAD3A patients 2, 3, and 4 (AGS1530, AGS2216, and AGS2792, respectively). Mean values and data points of 10-14 independent experiments are shown and are expressed as the ratio of the mRNA levels, normalized to housekeeping gene HPRT mRNA, in indicated conditions to that in one control HDF. ${ }^{*}$ indicates statistical significance $(P<0.05)$ in one-way ANOVA with Dunn's multiple comparison test. (B) Protein expression of ATAD3A in primary fibroblasts assessed by Western blot. VDAC1 is a mitochondrial protein, and vinculin a loading control. (C) qPCR analysis of mtDNA copy number per cell, expressed as the ratio of the DNA quantity of the mitochondrial gene (MT-COXII) over the nuclear gene (GAPDH), in total DNA isolated from fibroblasts. The mean of eight independent experiments is shown. ${ }^{*}$ indicates statistical significance $(P<0.05)$ in one-way ANOVA with Dunn's multiple comparison test. (D) Representative ISG (Mx1, RSAD2, OAS1, and IFI27), cGAS, and MAVS mRNA expression, expressed as in A, in control and ATAD3A patient-derived primary fibroblasts, after down-regulation of cGAS (sicGAS) or MAVS (siMAVS) by siRNA or treatment with control siRNA (siCTRL). Mean values and data points of six to nine independent experiments are shown. ${ }^{*}$ indicates statistical significance $(P<0.05)$ in two-way ANOVA with Dunnett's multiple comparison tests between siRNA conditions for each cell line. \# indicates statistical significance $(P<0.05)$ in two-way ANOVA with Dunnett's multiple comparison test between control and patient-derived fibroblasts for each siRNA condition. (E) Representative ISG (IFITI and MxI) and IFNb mRNA expression, expressed as in A, in control (HDF; three lines pooled) and ATAD3A patient-derived primary fibroblasts (Pt; three lines pooled), UT) or mtDNA-depleted by ddC as in Fig. $4 \mathrm{D}$, and stimulated with $1 \mu \mathrm{g} / \mathrm{ml} \mathrm{HT}$-DNA or lipofectamine (Lipo) alone for $24 \mathrm{~h}$. Mean values and data points of three independent experiments are shown. ns indicates nonsignificance in two-way ANOVA with Sidak's multiple comparison test between UT and ddC conditions. (F) Representative ISG (Mx1, OAS1, ISG15, and IFI27), ATAD3A, and TFAM mRNA expression, expressed as in A, in control and ATAD3A patient-derived primary fibroblasts, UT or mtDNA depleted by ddC as in Fig. 4 D. Mean values and data points of three to eight independent experiments are shown. ${ }^{*}$ indicates statistical significance $(P<0.05)$ in two-way ANOVA with Sidak's multiple comparison test between UT and ddC conditions. \# indicates statistical significance $(P<0.05)$ in two-way ANOVA with Dunnett's multiple comparison test between control and patient-derived fibroblasts for each treatment. (G) Representative ISG Mx1 and ATAD3A mRNA expression in fibroblasts vehicle-treated (DMSO) or treated with $300 \mu \mathrm{M}$ DIDS or $100 \mathrm{nM}$ rapamycin for $72 \mathrm{~h}$. Mean values and data points of five to seven independent experiments are shown. ${ }^{*}$ indicates statistical significance $(P<0.05)$ in two-way ANOVA with Sidak's multiple comparison test between treatment conditions. \# indicates statistical significance $(\mathrm{P}<0.05)$ in two-way ANOVA with Dunnett's multiple comparison test between control and patientderived fibroblasts for each treatment. (H) ISG mRNA expression, represented as an ISG score, and BAK1 mRNA expression expressed as in A, in fibroblasts after down-regulation of BAX (siBAX) or BAK1 (siBAK1) or both (siBAX+siBAK1) by siRNA or treatment with control siRNA (siCTRL). Mean values and data points of two to three independent experiments are shown. ${ }^{*}$ indicates statistical significance $(P<0.05)$ in two-way ANOVA with Dunnett's multiple comparison tests between siRNA conditions for each cell line. \# indicates statistical significance $(P<0.05)$ in two-way ANOVA with Dunnett's multiple comparison test between control and patient-derived fibroblasts for each siRNA condition. (I) Protein BAK1, BAX, and ATAD3A levels in primary fibroblasts assessed by Western blot; VDAC1 is a mitochondrial protein, and vinculin and cofilin are loading controls. (J) ISG mRNA expression, represented as an ISG score, in fibroblasts after treatment with 50-200 $\mu$ M of BAX inhibitor peptide V5 (BAXi V5) for $72 \mathrm{~h}$. Mean values and data points of three to four independent experiments are shown. \# indicates statistical significance $(P<0.05)$ in two-way ANOVA with Dunnett's multiple comparison test between control and patient-derived fibroblasts for each treatment.

Provided online are two tables. Table S1 summarizes molecular, clinical, and IFN data relating to patients 1-7. Table S2 summarizes features related to systemic sclerosis in patients 2 and 3.

Lepelley et al.

Interferon signaling in a mitochondrial disease 